\title{
Particle accumulation structures in non-cylindrical liquid bridges under microgravity conditions
}

\author{
Paolo Capobianchi* and Marcello Lappa ${ }^{\dagger}$ \\ Department of Mechanical and Aerospace Engineering, \\ University of Strathclyde, James Weir Building, \\ 75 Montrose street, Glasgow G1 1XJ, UK.
}

\begin{abstract}
The emergence of Particle Accumulation Structures (PAS) in non-cylindrical liquid bridges (LB) is studied numerically for a high Prandtl number liquid considering microgravity conditions. Simulations are conducted in the framework of a finite-volume (Eulerian) approach with non-isodense particles being tracked using a Lagrangian, one-way coupling scheme. First, the threshold of the Marangoni-flow instability is determined as a function of the aspect ratio and the volume of liquid held between the supporting disks, thereafter, PAS formation is investigated for supercritical conditions. The overall approach is specifically conceived to provide details about the morphological evolution of these structures as the main control parameters are varied. For this reason a set of new notions and definitions (such as the linear extension of the PAS, its inner core radius and the area of the "petals" or "blades") are introduced to allow a precise quantification of a series of purely geometrical effects. Though the analysis is deliberately limited to illustrating the macroscopic patterning behavior and its relationship with the overarching factors, a new model is proposed to interpret the increased ability of slender (concave) liquid bridges to support the formation of PAS over extended ranges of values of the particle Stokes number. This model yet relies on essentially geometrical arguments, that is, the triadic relationship among the curvature of the free surface, the topology of fluid streamlines and particle mass effects.
\end{abstract}

\footnotetext{
* paolo.capobianchi@strath.ac.uk

$\dagger$ marcello.lappa@strath.ac.uk
} 


\section{INTRODUCTION}

Various fundamental types of particle-fluid interaction stemming from different situations in nature and technology are often regarded as archetypal systems for advancing our knowledge of complex fluids and multiphase flows [1-17]. In such a context, the structures produced by the self-organization of particles have enjoyed a widespread interest over recent years as a classical workhorse to predict the nonlinear dynamics of these systems and their asymptotic behaviors. Though these situations differ in regard to the spatio-temporal nature of the flow (laminar or turbulent), and/or with respect to the physical effect driving it (a pressure difference or a force of buoyant, vibrational, or thermocapillary origin), the identification of general principles and overarching common mechanisms has actively been sought.

The present work, in particular, aligns with the line of inquiry originating from the landmark experiments carried out in liquid bridges (LB) by [18] and [19]. If the size of the fluid domain is relatively limited (few millimetres in normal gravity conditions) or the experiments are conducted in space, the dominant mechanism of convection in this configuration (a small amount of liquid suspended between two circular supports at different temperature) is generally represented by surface-tension-driven (Marangoni) convection. The typical hierarchy of bifurcations displayed by these systems is as follows: initially (for relatively small values of the imposed temperature difference) a unique flow structure is allowed, namely a steady and axisymmetric toroidal roll. On increasing the magnitude of the temperature gradient, however, this uniqueness is lost and other flows with well-defined different patterns of symmetry are established after transients have decayed away ([20] and references therein). In general, the initially steady convection is taken over by motions that are spatially periodic, quasi-periodic or chaotic [21-24].

The interest in this problem is still alive as witnessed by the studies being produced nowadays [25, 26].

The onset of time-dependence is generally associated with the emergence of waves which travel in the fluid with an axial component (directed in the upstream direction) and a symmetry breaking component oriented in the azimuthal direction. Due to the cylindrical symmetry of the system, these oblique waves are always produced in couples. Accordingly, 
two types of waveforms are allowed in principle, i.e. standing waves (resulting from the superposition of waves with equal amplitude) and traveling waves (waves with different amplitude, [27] and references therein). These modes of convection have effectively been observed in a number of experimental and numerical studies on the subject $[2,21]$.

After some initial observations by [28] where some glimpses of these peculiar particle phenomena were provided, the aforementioned [18] and [19] were the first to clearly report on the surprising emergence of fascinating collections of particles (perfectly aligned in space along paths with the topology of a multi-blade circuit or windmill). These phenomena (today generally referred to with the acronym of PAS, i.e. particle accumulation structures) manifest themselves in the presence of a traveling wave and in relatively restricted ranges of the imposed temperature difference and particle size. An extended description of their geometrical properties can be found in various papers appearing on the subject over the last decade. As an example, [19] described them as a circuit spirally wrapped around the core of the basic toroidal vortex. Other researchers could discern that the apparent property of this circuit to rotate in the azimuthal direction with the same angular frequency of the traveling wave (as it were a solid body), is just an illusory effect. Indeed, as demonstrated by [18], if examined individually, particles may even move in an opposite direction with respect to the prevailing sense of rotation of the wave (it has been clarified that a spatially rotating item with fixed shape appears because a relatively large number of particles undergo spatially correlated large-scale motions).

Research conducted over subsequent years involved sifting through existing (experimental, theoretical and numerical) approaches with different foci in order to glean hints and draw inferences about what factors may feed the mechanisms at the root of these structures [29, 30] These efforts led to the elaboration of two main theories running in parallel in the literature, relying on the concepts of 'inertial' or 'finite-size' Lagrangian coherent structures, respectively [3-5, 31-33]; [34-36].

These models have displayed a varying degree of success in explaining experimental dynamics depending on the considered circumstances.

Paralleling these continued theoretical efforts has been a series of other works, aimed to explore qualitatively and quantitatively different parts of the parameter space to reveal the intrinsic multiple nature of the solutions (particle attractors) which can characterize this problem. While some properties of these structures are inherited from the underlying flow 
pattern (the number of blades or 'petals' characterizing the projection of the particle circuit on a plane perpendicular to the liquid bridge axis generally reflects the so-called azimuthal wavenumber of the traveling wave, i.e. the number of sinusoidal distortions affecting the toroidal roll in the circumferential direction), other topological properties can display a varying nature, which has puzzled the investigators for years [6, 8, 9, 18, 37-39].

Along these lines, as an example, it is also worth mentioning that other researchers ([18], [34], and [40] and [41]) have reported on the existence of an alternate, less ordered state, generally referred to as the "toroidal core" given the tendency of particles to cluster where the fluid vorticity takes relatively high values. Romano and Kuhlmann [36] could show numerically that this peculiar behavior should be regarded as an intermediate stage of evolution. Oba et al. [41] indicated experimentally that the particles on the core exhibit chaotic motions. Lappa [42] found that the application of high-frequency high-amplitude vibrations along the axis of the liquid bridge could make this peculiar state stable.

Some studies have also demonstrated that different types of highly ordered PAS structures may co-exist in the same regions of the space of parameters [7, 10, 35, 37] and that asymmetric variants of PAS, i.e. windmills that display an eccentricity with respect to the axis of the liquid bridge, are possible $[10,43]$. Such line of inquiry has progressed with the aid and support of both experimental results and numerical simulations, which have shown that the aforementioned set of influential conditions can encompass a wide range of factors such as (but not limited to) the size of particles [36], their density [7, 19] or 'initial position' [10], the mono- or multi-dispersed nature of the dispersion [7], and the properties of the carrier flow (Marangoni convection) as determined by the value of the Marangoni number and the aspect ratio of the related toroidal vortex $[9,37]$. A similar concept also applies to the influence of the volume of liquid effectively held between the two disks supporting the liquid bridge for a fixed geometrical aspect ratio. However, only a few experiments have appeared in the literature dealing with this aspect $[8,44]$. Unfortunately, numerical results are completely absent due to the difficulties typically associated with the numerical simulation of non-cylindrical geometries.

In such a context, the present analysis should be seen as a very focused effort aimed to explore aspects which have been poorly addressed in the literature or completely overlooked. Specific numerical examples about non-cylindrical liquid bridges are conceived and examined towards the end to gain an increased understanding of the properties of the emerging particle 


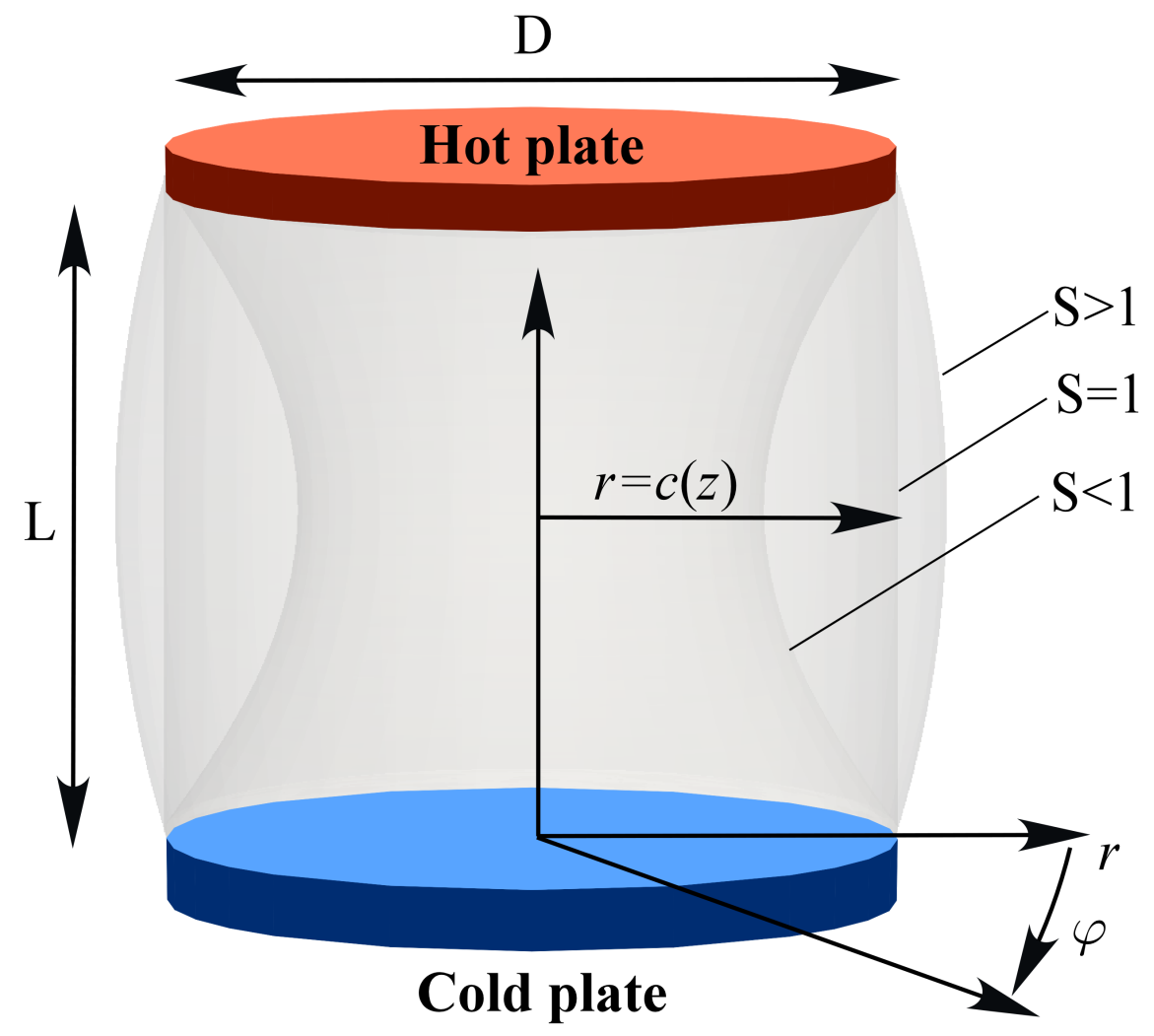

FIG. 1: Sketch of liquid bridges with different volume ratios, $S$, and the coordinates system adopted to represent the interface.

structures and the underlying fluid flow mechanisms.

\section{MATHEMATICAL AND NUMERICAL MODEL}

\section{A. Geometrical model}

The geometry of the problem is shown in Fig. 1. The liquid bridge is held by surface tension between two coaxial cylindrical disks (or rods) of diameter $D$, placed at a distance $L$ apart. The upper and lower rods are assumed to be flat and are kept at a different temperature (resulting in an imposed temperature difference, $\Delta T=T_{\text {hot }}-T_{\text {cold }}$ ). The free surface (interface separating the liquid from the external gaseous environment) is considered adiabatic. The liquid bridges aspect ratio is defined as $A R=L / D$. Gravitational effects are neglected (microgravity conditions).

The different variants shown in Fig. 1 essentially result from the different volume of 
liquid $(V)$, which can effectively be held between the two disks. Injecting in the liquid bridge an amount of liquid larger than that required to fill a cylinder with the same radius of the rods and height equal to the distance between them $\left(V_{0}\right)$ results in a convex interface $\left(V>V_{0}\right)$, whereas the opposite situation in which the liquid bridge is under-filled $\left(V<V_{0}\right)$ leads to a concave interface. A relevant non-dimensional parameter able to account for these situation is the volume ratio defined as $S=V / V_{0}[45] ;$ [46] and [47]. Another relevant parameter is the Capillary number, defined as, $C a=\sigma_{T} \Delta T / \sigma_{0}$, where $\sigma_{0}$ is the surface tension evaluated for a reference temperature $T_{0}$ and $\sigma_{T}$ is the surface tension temperature coefficient (formally linking the surface tension to the temperature via the linear relationship $\left.\sigma=\sigma_{0}-\sigma_{T}\left(T-T_{0}\right)\right)$. If this non-dimensional number is sufficiently small, the deformation of the free surface of the liquid bridge induced by the Marangoni flow can be neglected. Accordingly, the interface can be assumed to be rigid and its shape can be determined solving the Young-Laplace equation

$$
\Delta p+\rho g z=\sigma\left(\frac{1}{R_{1}}+\frac{1}{R_{2}}\right)
$$

where $\Delta p$ is the pressure jump across the two phases and $R_{1}, R_{2}$ are the principal radii of curvature. Equation 1 can be reformulated in non-dimensional form in cylindrical coordinates (cf. Fig. 1) by substituting the analytical expression of the principal radii of curvature $([46],[47])$. This leads to

$$
\frac{1}{c\left[1+\left(c^{\prime}\right)^{2}\right]}-\frac{c^{\prime}}{c\left[1+\left(c^{\prime \prime}\right)^{2}\right]^{3 / 2}}=\frac{L \Delta p}{\sigma_{0}}=k_{1}
$$

where $c=c(z)$ is the non-dimensional (with respect to $L$ ) radial position of the interface and the "primes" represent derivation with respect to the axial coordinate $z$. Each value of the parameter $k_{1}=L \Delta p / \sigma_{0}$ determines a fixed volume of the liquid bridges. In the present work, we have changed parametrically the value of $k_{1}$, in order to obtain the desired interface shape. The equation has been integrated by a shooting method with the conditions that the liquid is attached to the solid supports: $c=c(0)=c(1)=R / L$. 


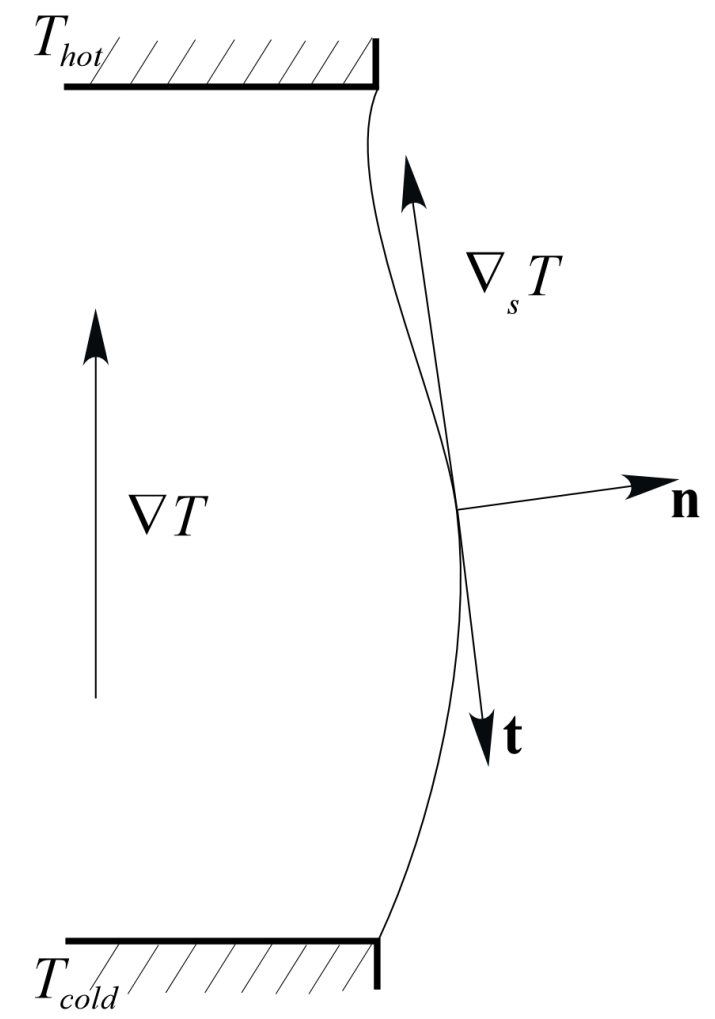

FIG. 2: Schematics of the liquid bridge interface showing the unit normal and tangent vectors, and the interface temperature gradient $\nabla_{s} T$.

\section{B. Fluid motion governing equations}

The equations governing the flow motion are the incompressible Navier-Stokes and energy equations, which in dimensionless form can be cast in compact form as (see, e.g., [48])

$$
\begin{aligned}
& \nabla \cdot \mathbf{u}=0, \\
& \frac{D \mathbf{u}}{D t}=-\nabla p+\nabla^{2} \mathbf{u}, \\
& \frac{D T}{D t}=\frac{1}{P r} \nabla^{2} T .
\end{aligned}
$$

Where $t$ represents time, $\mathbf{u}$ is the fluid velocity, and the operator $D / D t=\partial / \partial t+\mathbf{u}$. $\nabla$ indicates the substantial derivative (related reference quantities used to put the set of equations in non-dimensional form are $L, \nu / L, L^{2} / \nu, \rho_{0}(\nu / L)^{2}$ and $\Delta T$ for length, velocity, time, pressure and temperature, respectively, where $\nu$ is the fluid kinematic viscosity).

Closure of the mathematical problem also requires the definition of the conditions at the boundary of the liquid bridge. Neglecting the presence of the surrounding fluid, i.e. by 
considering the adiabatic interface as an impermeable rigid boundary where the thermocapillary action is balanced by the tangential viscous stress produced in the liquid, the related dynamic boundary condition at the free interface reads [48]:

$$
\left[\nabla \mathbf{u}+(\nabla \mathbf{u})^{\mathrm{T}}\right] \cdot \mathbf{n}+\operatorname{Re} \nabla_{s} T=0
$$

where $\nabla_{s} \equiv \nabla-\mathbf{n}(\mathbf{n} \cdot \nabla)$ and $\mathbf{n}$ is the unit normal vector at the interface (cf. Fig. 2). The dimensionless numbers appearing in the governing equations and stress boundary condition (Eqs. 3-6) are the Prandtl number, $\operatorname{Pr}=\nu / \alpha$, and the Reynolds number $\operatorname{Re}=$ $\sigma_{T} \Delta T L / \rho_{0} \nu^{2}$, where $\alpha$ is the thermal diffusivity of the fluid. Following common practice, in the reminder of the paper, we refer to the Marangoni number, formally given by the product of the Prandtl and Reynolds numbers, i.e. $M a=\operatorname{Re} \operatorname{Pr}=\sigma_{T} \Delta T L / \rho_{0} \nu \alpha$.

\section{Particle dynamics}

The dynamics of finite size, non-massless particles are generally different from the behavior of the fluid in which they are dispersed in. Furthermore, in certain conditions, dispersed solid particles can even have a back influence on the local flow field (making flow and particles dynamics essentially 'coupled) or interact reciprocally (e.g., particle-particle collision). For relatively limited dimension of the tracers, particle Stokes number of the order of $10^{-5}$ or lower (see e.g., [19]), and dilute dispersions (the ratio of particles volume to liquid bridge volume is of the order of $10^{-4}$ in typical experiments, see e.g., [19]), such coupling effects are generally neglected, and the simulations are conducted on the basis of the so-called one-way coupling approach. In other terms, the flow field is assumed to be completely insensitive to the presence of the dispersed phase.

Though this numerical approach should clearly be regarded as a simplification of reality (obviously this scheme cannot describe particle interaction when accumulation takes place), it has enjoyed a widespread use in the literature for the study of PAS ([3-5], [10], [29], [34-36], [43], [48]) . A justification or rationale for such a practice can be found in available experimental observations or data ([2], [6-9], [19], [37-41], [44]). Indeed, experiments dealing with a variety of fluids and particle types have shown that once particles enter the attractor

(the KAM torus), they can no longer leave it (inter-particle forces are not sufficiently strong to allow particles to get away from the attractor). 
In the present work, particles are tracked according to the Maxey-Riley equation (see, e.g., [49]), which is reported below in dimensionless form

$$
\frac{d \mathbf{v}}{d t}=\frac{1}{\xi+1 / 2}\left[\frac{3}{2} \frac{D \mathbf{u}}{D t}-S t^{-1}(\mathbf{v}-\mathbf{u})\right]
$$

The two terms appearing in the brackets present at the right hand side of Eq. 7 are, in order of appearance, the pressure gradient force and the drag force. The factor present at the denominator outside the brackets takes into account the added mass force. The parameter $\xi=\rho_{p} / \rho$ represents the particle-to-fluid density ratio. On writing Eq. 7, the Saffman and Faxén corrections have been neglected, as usual in these problems (see e.g., [36]).

The additional dimensionless group appearing in this equation is the particle Stokes number defined as, $S t=d_{p}^{2} / 18 L^{2}$, where $d_{p}$ is the aforementioned particle diameter.

The Basset force has also been neglected, which requires proper justification. Some relevant information along these lines can be found in the work by [42]) where related arguments were elaborated on the basis of the earlier studies by [50] and [51] about the motion of spherical particles in turbulent fluids and harmonic stokes flows, respectively, and by [52] about history effects in high-frequency flows. As shown by these studies, the Basset force can be ignored provided particles and the oscillatory motion induced by an imposed external forcing (e.g. vibrations or g-jitters in microgravity conditions) or spontaneously produced by the considered flow (as a result of a Hopf bifurcation) satisfy well-defined conditions.

These conditions are in general formulated directly in terms of $S t$ and the additional parameter (see, e.g. [51]) defined as $S t_{\omega}=\omega_{\text {dim }} d_{p}^{2} / 18 \nu=\omega S t$ where $\omega_{\text {dim }}$ is the frequency of oscillation of the velocity field $\left(\omega=2 \pi f_{c}\right)$. General consensus exists that the relative importance of the Basset force increases with both $\xi$ (particle-to-fluid density ratio) and the non-dimensional $\omega$. In particular, according to the criteria provided by [50], the effect of the Basset force should be neglected a priori when the Stokes number $(S t)$ satisfies the condition $S t<2 / 9 \xi^{1 / 2}\left(\cong 3 \times 10^{-1}\right.$ for $\xi=2$ and $\cong 1.5 \times 10^{-1}$ for $\left.\xi=0.5\right)$. An evaluation of $S t$ and $S t_{\omega}$ with the present choice of parameters (see Sect. III) shows that this criterion is met with $S t \cong 10^{-4}$ and $S t_{\omega} \cong 5.6^{-2}$ (considering for $\omega$ the maximum possible value, i.e. the "worst" case $\omega \cong 70$, the reader being referred to the plots about the frequency of the oscillatory flow reported in Sect. III C).

Assuming a density ratio larger than those considered here $(\xi=2.7)$, [53] concluded that 
the effect of the Basset term could be neglected, provided the nondimensional angular frequency $\omega$ of the sinusoidally varying velocity field was smaller than $6.2 \times 10^{2}(<0.1$ with their reference units, see Figure $2 \mathrm{a}$ of their work). These conclusions also agree with the analysis by [54] and [51]. In our case this condition is also satisfied.

The latter authors, in particular, found that even though the importance of the Basset term decreases rapidly as the value of $S t_{\omega} \rightarrow 0$ for all values of $\xi$ (as also confirmed later in the experiment by [52]), however, the ratio of the Basset force to the overall force experienced by the particle (virtual mass + stokes drag + history term) is $\cong 0.2$ (see Figure 1 of their work) for a value of their control parameter " $S$ " $=0.6$, which would correspond in the present case to a frequency of oscillation $\omega \cong 1.25 \times 10^{3}$.

Put together, all these studies essentially indicate that the Basset term may play a nonnegligible role for the conditions considered in the present study only when $\omega>10^{3}$. As evident in the figures reported in Sect. III C, the frequencies relating to the present cases are much smaller than this limit. The influence of the Basset force can therefore be considered negligible.

\section{Solution Method}

Equations 3-7 have been solved in the present work using the OpenFoam computational platform. In particular, the Navier-Stokes equations have been solved in the frame of the well-known PISO approach [55].

With OpenFoam such algorithm relies on a collocated grid arrangement of primitive variables, that is, all velocity components, pressure and temperature are defined in the center of each control volume. Relevant coupling of all these variables (needed to avoid the onset of numerical oscillations and keep the time-marching procedure stable) is ensured through a special interpolation scheme implemented on the faces of the volumes [56].

In particular, in the present study, the different diffusive and convective terms appearing in Eq. 4 and Eq. 5 have been discretized using second order central difference schemes. A Preconditioned Bi-Conjugate Gradient (PBiCG) together with an Incomplete Lower Upper (DILU) preconditioner in the predictor step has been used to treat the resulting system of algebraic equations. The additional pressure equation of elliptic nature associated with the PISO has been iteratively integrated in the frame of a Generalized Geometric-Algebraic 
Multi-Grid (GAMG) method (the reader being referred to Sect. III A for additional information about the solver validation and the grid refinement study).

\section{E. Particle-boundaries interaction}

A separate discussion is needed for the specific algorithm used to track particle motion. To ensure high accuracy, the Maxey-Riley equation has been integrated with a time step satisfying the following restricive condition: maximum particle Courant number $\sim 10^{-3}$.

Moreover, specific attention has also been paid to the particle-wall interaction model.

In this regard, OpenFOAM offers a variety of different options. The present work relies on the so-called local interaction model. In particular, we have implemented it in order to modify the component of the particle velocity perpendicular to the wall (as the particle approaches the boundary) while keeping unchanged the original tangential velocity.

Put simply, when the particle is localized inside a cell adjacent to a boundary, the algorithm makes a check on the normal velocity and, if this value is positive (meaning that the particle is moving toward the boundary), the particle-wall interaction model is activated in order to modify the velocity according to the following relationship (Eq. 8)

$$
\begin{cases}{[\mathbf{u}]_{h+1}=[\mathbf{u}]_{h}-\left\{\left(1+e_{p}\right)\left[u_{\mathbf{n}}\right]_{h} \mathbf{n}+\mu_{p}\left[u_{\mathbf{t}}\right]_{h}\right\},} & \text { if } u_{\mathbf{n}}>0 \\ {[\mathbf{u}]_{h+1}=[\mathbf{u}]_{h},} & \text { otherwise }\end{cases}
$$

where $h$ is the time step at which the particle hits the wall and the $h+1$ is the subsequent one. $u_{\mathbf{n}}, u_{\mathbf{t}}$ represent the component of the velocity normal and tangential to the cell face, being the interface or a solid wall, indistinctly.

To take into account the effective physical extension of particles and avoid that the distance between their center and the boundary of the considered physical domain becomes smaller than the particle radius (e.g., situation corresponding to the unphysical penetration of particles into the walls or the interface), we have modified the model under discussion to prevent the particle spherical surface from crossing the external boundaries of the liquid bridge.

All the simulations discussed in the reminder of this paper have been conducted by setting $e_{p}=\mu_{p}=0$, therefore the particle approaching the wall will proceed tangentially to the wall itself (the velocity perpendicular to the wall is annihilated, while the tangential one is 


\section{Computational cell adjacent to a boundary}

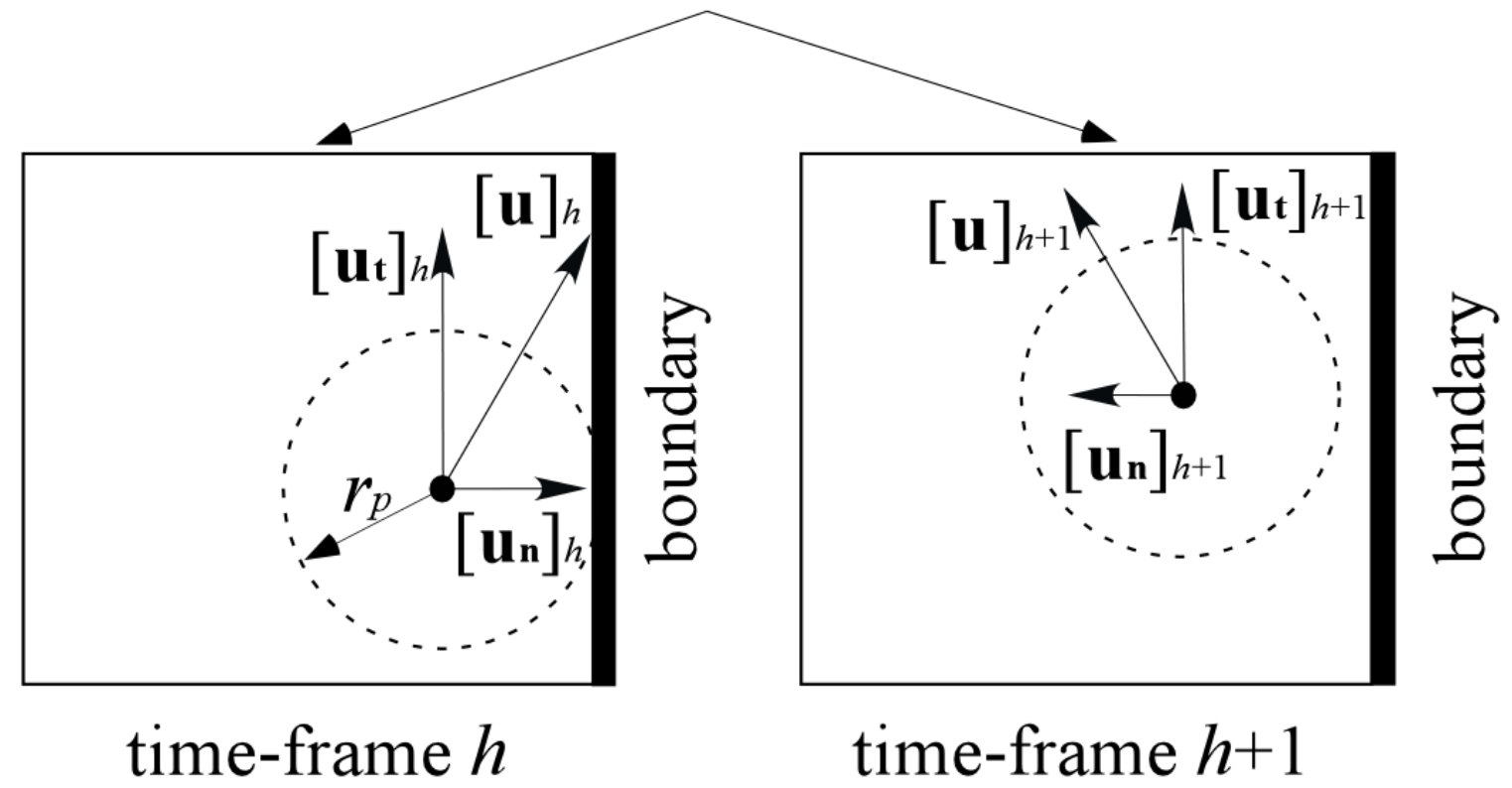

FIG. 3: Computational cell next to a boundary showing the particle velocity before "time-frame $h ")$, and after "time-frame $h+1 ")$ the particle-boundary interaction treatment.

conserved).

Similar approaches have been adopted successfully in previous studies by (see, e.g., [36]).

\section{RESULTS}

Most of results have been obtained for $S \neq 1$ (although the cases where $S=1$ have also been examined for the sake of completeness and for comparison with the cases $S \neq 1$ ). Moreover, as explained in the earlier section, we have considered microgravity and perfectly rigid interfaces under the assumption that $C a \ll 1$.

To the best of our knowledge the experimental studies by [44] and [8] are the only works in which the effect of interface deformation on PAS formation was investigated (though under normal gravity conditions). Here the problem is addressed numerically with the two-fold intention to 1) study situations (microgravity) for which no experimental data are avail- 
able and 2) provide additional details and insights into the topological and morphological evolution of PAS when non-straight interfaces are considered.

\section{A. Code validation and Mesh study}

In order to test the capabilities of the solver to correctly capture the hydrothermal instability of Marangoni flow and, the ensuing ability of these flows to force dispersed particles to accumulate in coherent structures, we have initially considered the same numerical setup of [4] and [42]. The test case (see Table I) is a liquid bridge with unit volume ratio $(S=1)$ and aspect ratio, $A R=0.34$ in microgravity conditions. Sodium-nitrate $(\operatorname{Pr}=8)$ is assumed as the working fluid, while the Marangoni number is, $M a=20600$, which on the basis of our computations (see Sect. III B) corresponds to $M a / M a_{c} \approx 2.25$.

TABLE I: Numerical setup of the experiment for the case studied by [4], [42]

\begin{tabular}{lcccc}
\hline \hline Working fluid & $\operatorname{Pr}$ & $\nu\left[\mathrm{m}^{2} \mathrm{~s}^{-1}\right]$ & $\rho\left[\mathrm{kg} \mathrm{m}^{-3}\right]$ & $M a$ \\
\hline Sodium nitrate & 8 & $1.27 \times 10^{-6}$ & 1904 & 20600 \\
\hline \hline
\end{tabular}

Using a mesh with resolution similar to those considered by [4], [42] (a mesh with this density is shown in Fig. 4 for a liquid bridge with convex interface), we have found the flow to be unstable with the emergence of a hydrothermal traveling wave having wave number $m=3$. The angular frequency, $\omega=2 \pi / m \Theta$, where $\Theta$ is the period of the oscillations measured by a probe located at a fixed position inside the liquid bridge is in good agreement with the previous findings by [4], [42] with a relative percentage error, $\epsilon<2 \%$, as indicated in the last column of Table II.

As the next step of this validation hierarchy, we have considered the dynamics of solid particles (their number being $n_{p} \approx 1000$ ) through the solution of Eq. 7. The corresponding PAS reported in Fig. 5a closely resembles the PAS obtained by [42], (cf. Fig. 5b), thereby providing further evidence for the reliability of the present approach,

A grid assessment study to assess the sensitivity of the overall computational algorithm to the mesh has been conducted adopting two levels of refinement (obtained by increasing the number of points along each direction in the coordinate system, $(r, \varphi, z))$. The outcomes of such assessment are summarized in Table III in terms of values of the angular frequency 
Side view

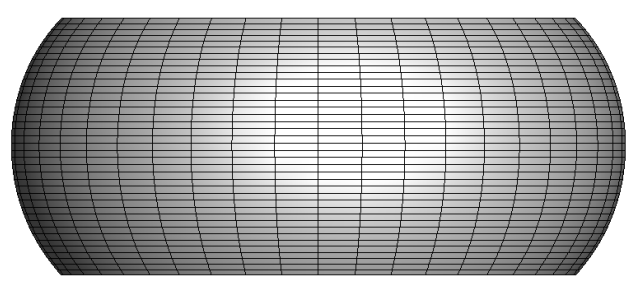

Top view

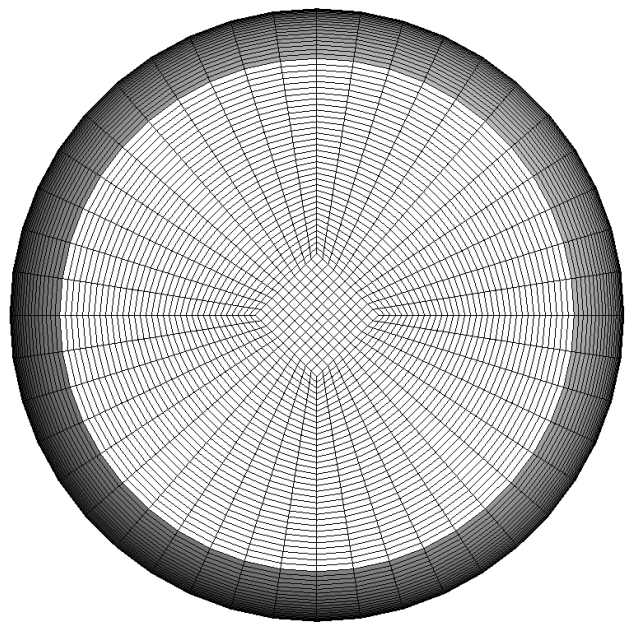

FIG. 4: Computational domain (mesh $M_{1}$ ) for the configuration $A R=0.34, S=1.1$

TABLE II: Code validation: comparison with the previous results by [4], [42].

\begin{tabular}{ccccc}
\hline \hline & Number of cells $(r, \varphi, z)$ & Mode $m$ & $\omega[H z]$ & PAS \\
\hline Melnikov et al., (2011) & $(40,32,40)$ & 3 & 2.81 & Yes \\
Lappa (2013) & $(40,40,32)$ & 3 & 2.80 & Yes \\
Current work & $(40,40,40)$ & 3 & 2.78 & Yes \\
\hline \hline
\end{tabular}

and related percentage error versus the density of the mesh.

Owing to the good rate of convergence displayed by the algorithm and the modest percentage error relating to a switch from the mesh $M_{1}$ to the $M_{2}$, all the simulations discussed in the reminder of this paper have been carried out using the resolution $M_{1}$ reported in Table III (which has proven to be the best compromise in terms of accuracy and computational efficiency). 


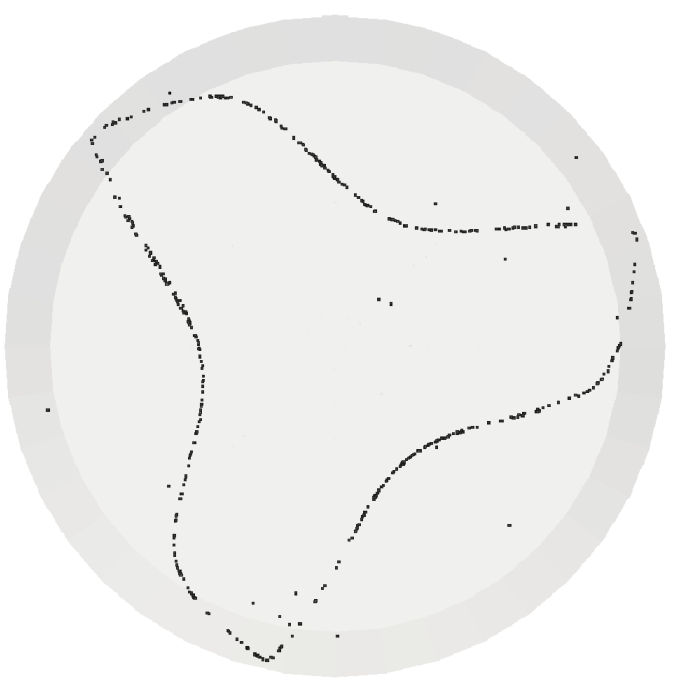

(a)

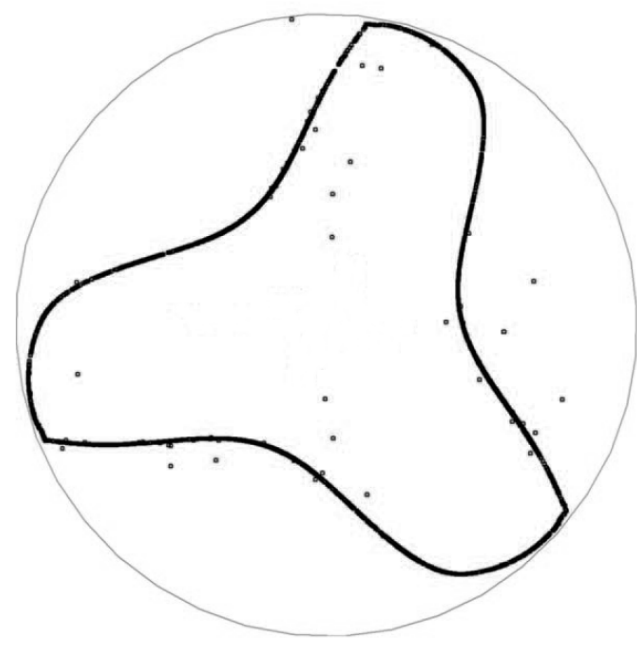

(b)

FIG. 5: Top view of PAS obtained obtained numerically: (a) present simulation, (b) after [42]. Flow conditions are summarized in Table I. (In frame (a), the larger circle represents the periphery of the closest disk, while the smaller circle is periphery of the opposite disk; the shaded region corresponds to the axial projection of the interface).

TABLE III: Outcome of the mesh convergence study. The three levels of refinement, $M_{1,2,3}$ have been obtained by increasing gradually the number of points along the three fundamental directions of the reference system

\begin{tabular}{cccc}
\hline \hline Mesh & Number of cells $(r, \varphi, z)$ & $\omega[H z]$ & $|\epsilon| \%$ \\
\hline$M_{1}$ & $(40,40,40)$ & 2.76 & 4.3 \\
$M_{2}$ & $(60,60,60)$ & 2.65 & 1.9 \\
$M_{3}$ & $(90,100,90)$ & 2.70 & \\
\hline \hline
\end{tabular}

B. Investigation of the critical conditions for liquid bridges with different aspect and volume ratios

As already explained in the introduction, particle accumulation in LB occurs only if the flow undergoes transition to a 3 -D oscillatory state and the emerging waveform is a 


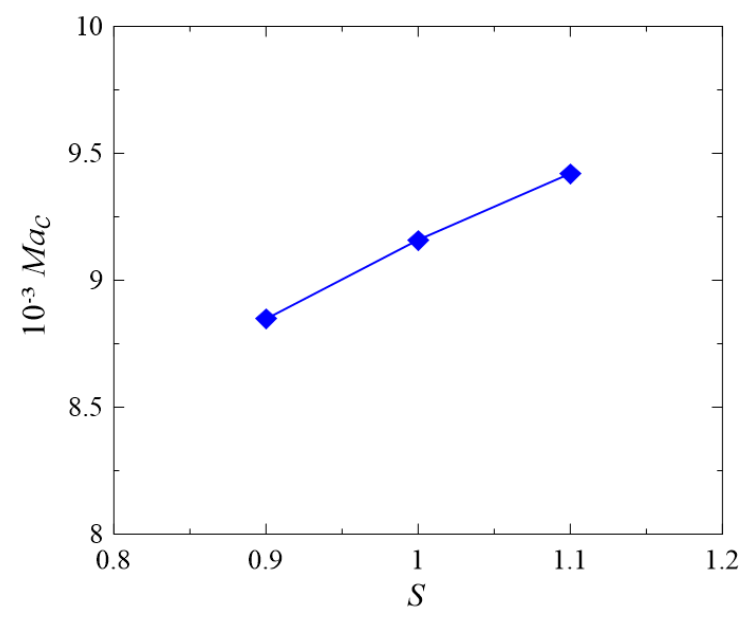

(a)

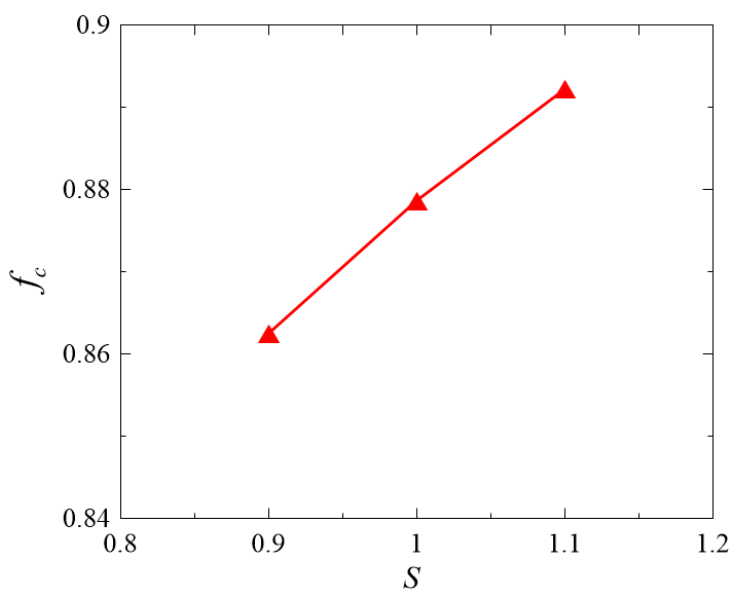

(b)

FIG. 6: Critical Marangoni number and respective critical frequency versus volume ratio for the cases with aspect ratio $A R=0.34$

traveling wave; hence, the Marangoni number has to be larger than the value at which the instability occurs (in the following indicated as the critical Marangoni number, $M a_{c}$ ). Specifically, according to previous observations (se e.g., [19]), particle accumulation is known to occur when the Marangoni number is such that $M a / M a_{c} \sim 2$. This simple argument implicitly indicates that a necessary pre-requisite for the analysis of particle behaviour is the determination of the critical conditions for all the LB configurations under investigation.

In the present work such a threshold has been determined through an "extrapolation" methodology (refer to, e.g., [57] for the details of the technique), i.e., by analysis of the rate of growth of the maximum azimuthal component of the velocity at the interface. This approach has been implemented in practice by considering two supercritical states relatively close in the parameter space, i.e., two subsequent values of the Marangoni number presumably located above the not-yet determined threshold. The critical values has finally been obtained via linear extrapolation to zero of the disturbance growth rate. More precisely, an iterative process has been implemented in which the ability of the system to produce an oscillatory response has been tested for progressively smaller values of the parameter, i.e. the Marangoni number. While doing so, we have assumed the fluid viscosity to be insensitive to temperature variations.

The data for $A R=0.34$ are reported in Fig. 6a, where the extrapolated $M a_{c}$ is shown for three different volume ratios: $S=0.92,1,1.1$. Fig. 6 b collects the corresponding values 


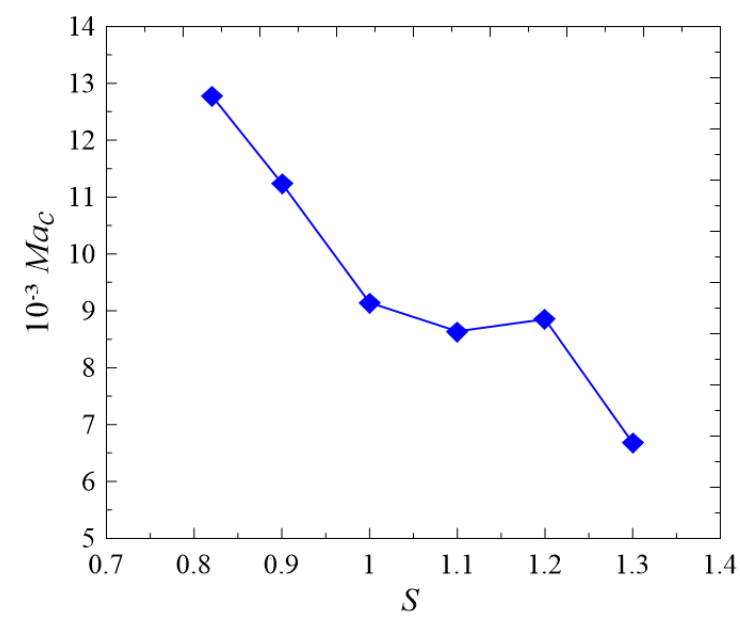

(a)

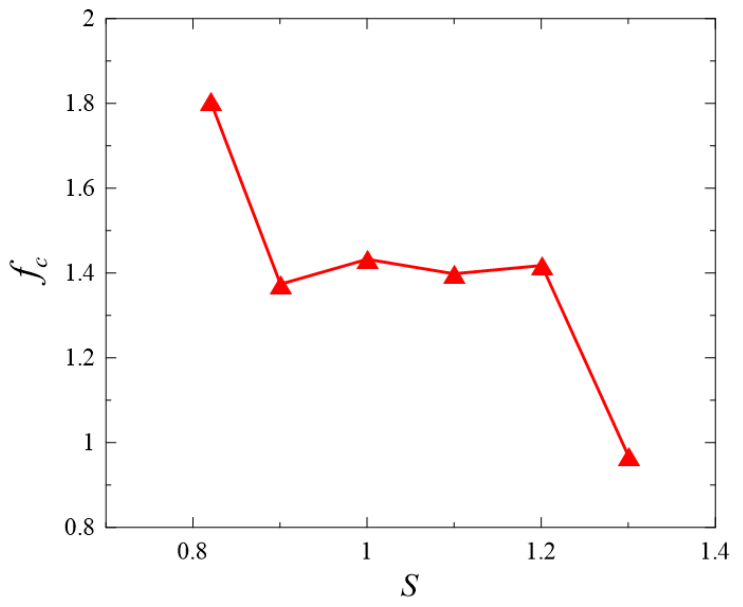

(b)

FIG. 7: Critical Marangoni number and respective critical frequency versus volume ratio for the cases with aspect ratio $A R=0.5$

of the critical frequency in dimensionless form, $f_{c}=H^{2} / \nu f$. It can be seen that both $M a_{c}$ and $f_{c}$ increase monotonically with $S$.

Fig. 7a,b summarize the critical Marangoni and frequency as a function of $S$ for $A R=0.5$. As witnessed by these figures, the trend is no longer monotone. $M a_{c}$ initially decreases, then a plateau region is attained $(1<S<1.2)$. After this region, a further decrease in $M a_{c}$ can be noticed. The frequency displays a similar behavior although the extension of the plateau region is larger.

For the highest aspect ratio considered $(A R=0.9)$ we have found significant (sudden) variations for the Marangoni number and related frequency (the affected ranges of $S$ being $0.8<S<0.9$ for $M a_{c}$, see Fig. 8a, and $0.9<S<1$ for $f_{c}$, see Fig. 8b). Such scenario, however, was not unexpected since similar results were predicted by earlier analyses.

The effect of volume $(S \neq 1)$ has indeed been studied by several other authors. As an example, through terrestrial experiments with transparent high-Prandtl liquids (various silicone oils), [58] and [59, 60] were the first to reveal the non-monotonic relationship between the stability limit and the liquid-bridge volume ratio i.e. these authors could clearly show that the stability diagram consists of two branches with an "overstability" gap (i.e. very high values of the critical Marangoni number) for intermediate values of the volume $(S \cong 0.9)$. In many of these experiments the overstability gap was found to correspond to $0.8<S<1.0$. These trends were confirmed by linear stability analyses, e.g., [61] for $\operatorname{Pr}=2,4$ and 7 and 


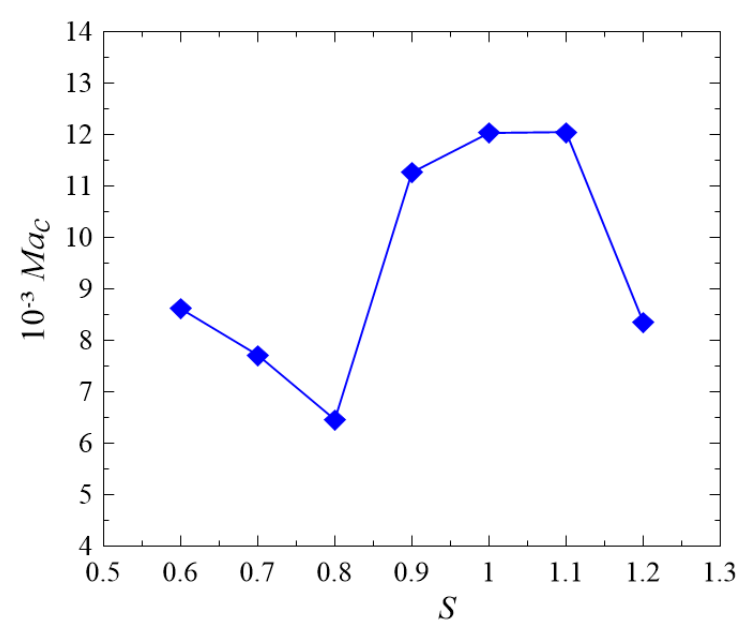

(a)

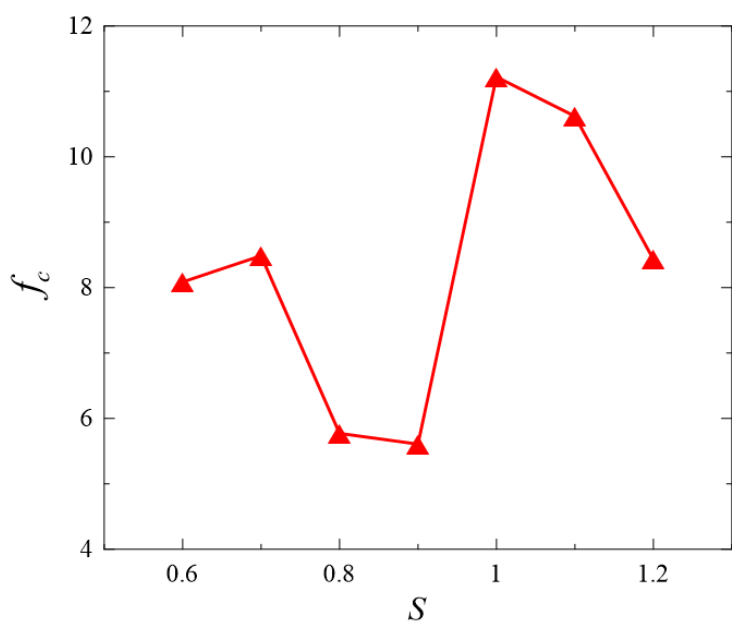

(b)

FIG. 8: Critical Marangoni number and respective critical frequency versus volume ratio for the cases with aspect ratio $A R=0.9$

$A R=0.5,[62])$ for a $5[\mathrm{cSt}]$ silicone oil, $0.5 \leq S \leq 1.4$ and $A R=0.75$, and numerical solution of the nonlinear thermal-convection equations (e.g., [63] for a 10 [cSt] silicone oil, $\operatorname{Pr}=105.6,0.6 \leq S \leq 1.1$ and $A R=0.8$ and [64] for $\operatorname{Pr}=27$ and $A R=0.77)$. A linear stability analysis for microgravity conditions was conducted by [65] for $\operatorname{Pr}=1,10$ and 50, a range of aspect ratios $0.4 \leq A R \leq 1.4$ for three values of $S=0.8,1$ and 1.2 and a wide range of nondimensional volumes $0.4 \leq S \leq 1.4$ for the particular case $\mathrm{AR}=0.6$. Interestingly, these authors reported that for $\operatorname{Pr}=10$ (a value relatively similar to that considered in the present work), a variation of $20 \%$ of the volume of the liquid bridge can lead to a variation of $100 \%$ of the critical Marangoni number; in particular, they found strong flow stabilization in the range $0.8<S<1$ (as also confirmed by available experiments, e.g., [60]), which is in very good qualitative agreement with the behaviour shown in Fig. 8 (the reader being also referred to the very similar plots reported by [62]).

Figure 9 provides the contours of the dimensionless temperature distribution (evaluated as $T^{*}=\left(T-T_{0}\right) / \Delta T$, where $T_{0}=\left(T_{\text {hot }}+T_{\text {cold }}\right) / 2$ is the average temperature $)$ in a meridian plane of the LB, and in the mid-plane perpendicular to the axis for some representative cases (flow conditions for each case are indicated in the caption). 

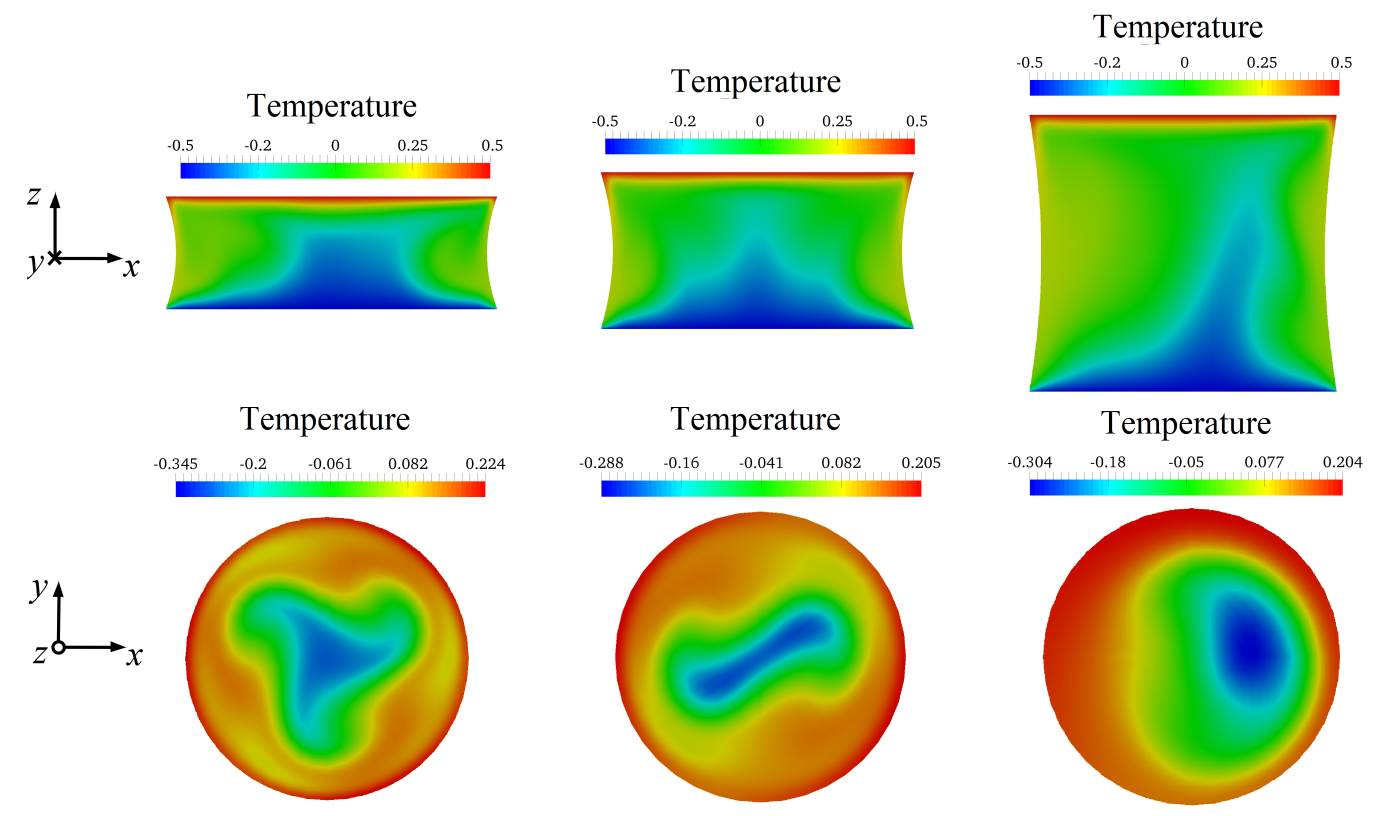

FIG. 9: Meridian plane (top), and axial view (below) of the temperature field in the presence of hydrothermal waves for three different aspect ratios: $A R=0.34, S=0.92$ (left), $A R=0.5, S=0.82$ (center), and $A R=0.9, S=0.8$ (right). The periphery of the circular cross sections corresponds to the free surface of the liquid bridge.

\section{Particle accumulation in deformed liquid bridges}

\section{Investigation of the region of existence of $P A S$}

In the earlier section, critical conditions for the onset of oscillatory flow (Hopf bifurcation) have been determined for liquid bridges with different aspect and volume ratios. Building on those results, in the present section, we investigate the circumstances under which PAS can emerge. In such a context we focus expressly on situations where particle mass is likely to play a central role in the process leading to structure formation (i.e. cases where $\xi \neq 1$ and for which there is an appreciable gap of density between the particles and the hosting fluid, that is $\xi \approx 2$ and $\xi=0.5$ ) and concentrate on situations with $M a / M a_{c} \sim 2$. As already explained to a certain extent in the introduction, rather than seeking mathematical answers to the mechanisms driving the formation of these structures (for which there are already running lines of research in the literature and an ongoing debate), we examine purely topological and morphological aspects and consider configurations for which new 

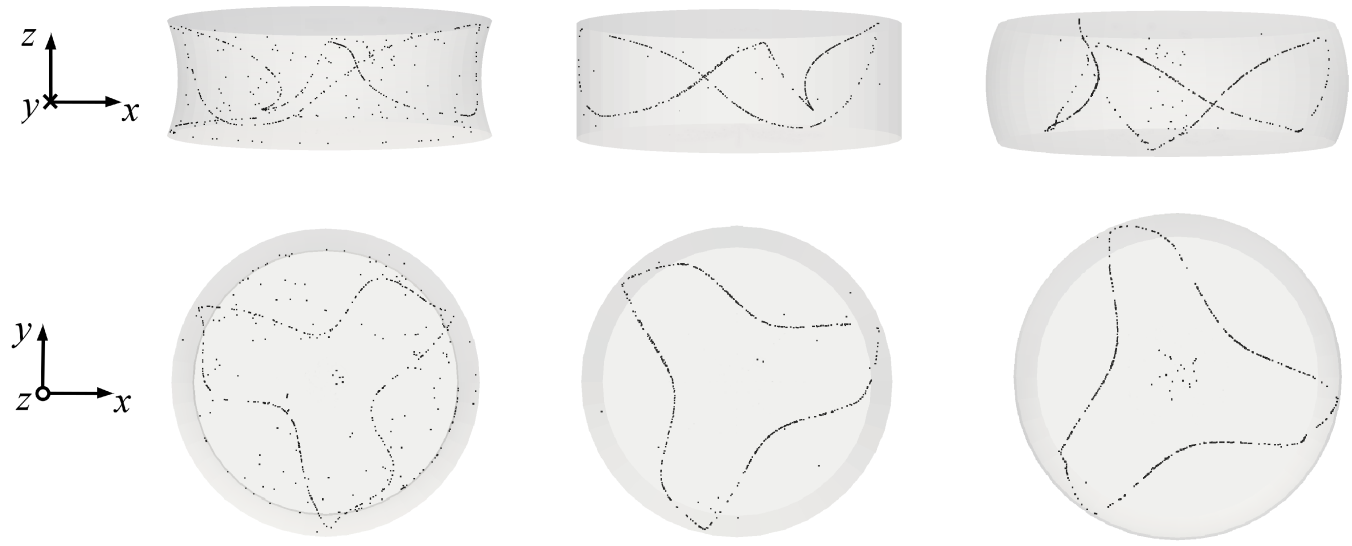

FIG. 10: Lateral and top views of PAS obtained numerically for the case $A R=0.34$ and three different volume ratios: $S=0.92\left(t^{*} \approx 0.375\right)$ (left), $S=1\left(t^{*} \approx 2.5\right)$

(center), and $S=1.1\left(t^{*} \approx 0.88\right)$ (right). Flow conditions are summarized in Table IV. (For the axial views, the larger circle represents the periphery of the closest disk, while the smaller circle is the periphery of the opposite disk; the shaded region corresponds to the axial projection of the interface). The same perspective mode has also been used for other similar 3D figures included in the present work.

particle structures may show up.

In particular, following a logical process, with situations of increasing complexity being examined as the discussion progresses, we begin our analysis from the same aspect ratio already considered for the validation study, that is, the liquid bridge with $A R=0.34$ (already examined by [4], [42] and many other authors).

We wish to recall that our criterion for judging on the attainment of a fully developed flow has been based on the observation of the signal (temperature) produced by a probe located near the interface. These observations were carried out over at least a dozen of subsequent wave peaks. When the period and amplitude percentage difference between two subsequent peaks was found to be less than $1 \%$, we judged the flow to be fully established. Subsequently, particles were tracked starting from the very last time frame available from the flow simulations until a well formed PAS could be observed. We considered the PAS completely formed where no changes could be seen in its morphology by prolonging the simulation over other 10 revolution periods.

Along these lines, Fig. 10 shows the side and top views of the emerging PAS for the cases 
$S=0.92$ (left view) and 1.1 (right view), respectively (to be directly compared with the structure obtained for $S=1)$ taken at different dimensionless time instants, $t^{*}=t /\left(L^{2} / \nu\right)$. The flow parameters for each simulation are summarized in Table IV. As a fleeting glimpse into this table would confirm, for this aspect ratio the wavenumber $(m)$ is insensitive to volume variations. Since the influence of $m$ is naturally filtered out, this should be regarded as a very suitable condition to evaluate the influence of the volume on the geometrical properties of the PAS.

Along these lines, by inspecting Fig. 10, the reader will easily realize that, from a purely qualitative standpoint, the main features of the PAS remain essentially unvaried, i.e. a tri-lobe structure $m=3$ PAS can be recognized for each case. Nevertheless, significant topological modifications can be spotted, especially near the deformed interface.

More pronounced variations can be seen especially for the configurations $S=0.92$. In this case the PAS appears significantly deformed with respect to the cylindrical and convex setups (cf. the top views shown in Fig. 10).

We argue that such modifications essentially reflect the corresponding radial expansion or contraction undergone by the toroidal Marangoni vortex (the reader being also referred to the related considerations elaborated by [46]). This aspect, as well as other morphological variations will be thoroughly described in the forthcoming section where we will introduce a set of "measures" especially conceived for a quantitative characterization of them.

In this section, rather we concentrate on findings about the effect of the particle Stokes number and density ratio yet for $A R=0.34$ (Table $\mathrm{V}$ ). These results are particularly interesting as they provide meaningful information in terms of "basin of attraction" (i.e. the region of existence of the considered phenomena in the space of parameters) and confirm (in line with the existing literature) that both $\xi$ and $S t$ are relevant influential factors. In particular, Table $\mathrm{V}$ is instrumental in showing that liquid bridges with concave interface $(S=0.92)$ are more prone to support the emergence of PAS. This is clearly witnessed by cross comparison of the outcomes of simulations conducted for the same Stokes number, density ratio and volumes smaller or larger than the cylindrical one. Notably, the same conclusions are also supported by Table VI where we have collected the analogous results for $A R=0.5$ (we will come back to this important observation later).

The particle structures for $A R=0.5$ are shown in Fig.11 for three representative values of the volume ratio $S=0.82,1,1.3$. Also in this case, in the considered range of values 
TABLE IV: Flow conditions for particle accumulation in liquid bridges with aspect ratio

$$
A R=0.34
$$

\begin{tabular}{cccccc}
\hline \hline$S$ & $M a / M a_{c}$ & $m$ & $S t$ & $\xi$ & PAS \\
\hline 0.92 & 2.33 & 3 & $8.89 \times 10^{-5}$ & 1.85 & Yes \\
1.00 & 2.25 & 3 & $8.89 \times 10^{-5}$ & 1.85 & Yes \\
1.10 & 2.19 & 3 & $8.89 \times 10^{-5}$ & 1.85 & Yes \\
\hline \hline
\end{tabular}

TABLE V: Summary of the whole set of simulations carried out for the case $A R=0.34$ for different values of $S t$ and $\xi$. The outcome of each run is summarized in the last column

\begin{tabular}{ccccc}
\hline \hline$S$ & $M a / M a_{c}$ & $S t$ & $\xi$ & PAS \\
\hline 0.92 & 2.33 & $1.39 \times 10^{-5}$ & 1.85 & Yes \\
0.92 & 2.33 & $2.20 \times 10^{-5}$ & 2.35 & Yes \\
0.92 & 2.33 & $2.20 \times 10^{-5}$ & 1.85 & Yes \\
0.92 & 2.33 & $5.00 \times 10^{-5}$ & 1.85 & Yes \\
1.10 & 2.19 & $5.00 \times 10^{-5}$ & 1.85 & Yes \\
1.10 & 2.19 & $8.89 \times 10^{-5}$ & 0.50 & No \\
1.10 & 2.19 & $8.89 \times 10^{-5}$ & 2.0 & Yes \\
1.10 & 2.19 & $8.89 \times 10^{-5}$ & 2.35 & No \\
1.10 & 2.19 & $1.40 \times 10^{-4}$ & 1.85 & No \\
1.10 & 2.19 & $2.20 \times 10^{-5}$ & 1.85 & No \\
\hline \hline
\end{tabular}

of the Marangoni number, a modification of the volume does not produce a change in the azimuthal wavenumber (which takes a fixed value, $m=2$ ). Yet, the alterations induced in the morphology of the toroidal Marangoni roll are reflected by appreciable changes in the PAS. The transverse extension of the PAS scales with the volume. These observations are quantitatively substantiated by the numerical results (in particular, by Fig. 11, where it can clearly be seen that the PAS for $S=0.82$ is considerably narrower than the others).

It can therefore be concluded that the trend displayed by the PAS in terms of radial extension is the same for both $m=2$ and $m=3$. 
TABLE VI: Flow conditions for particle accumulation in liquid bridges of aspect ratio

\begin{tabular}{cccccc}
\multicolumn{5}{c}{$A R=0.5}$. \\
\hline \hline$S$ & $M a / M a_{c}$ & $m$ & $S t$ & $\xi$ & PAS \\
\hline 0.82 & 1.72 & 2 & $1.6 \times 10^{-5}$ & 1.85 & Yes \\
0.90 & 2.00 & 2 & $1.6 \times 10^{-5}$ & 1.85 & Yes \\
1.00 & 1.69 & 2 & $1.6 \times 10^{-5}$ & 1.85 & Yes \\
1.10 & 1.62 & 2 & - & - & No \\
1.20 & 2.03 & 2 (standing) & - & - & No \\
1.30 & 1.80 & 2 & $1.6 \times 10^{-5}$ & 1.85 & Yes \\
\hline \hline
\end{tabular}
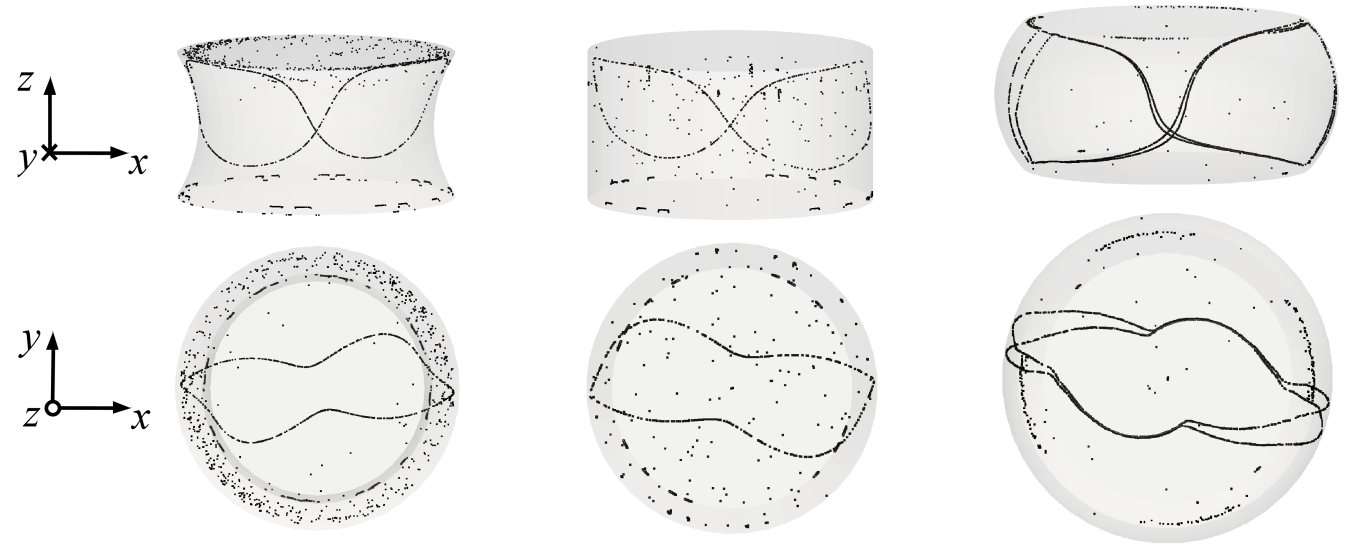

FIG. 11: Lateral and top views of PAS obtained numerically for the case $A R=0.5$ and three different volume ratios: $S=0.82\left(t^{*} \approx 0.16\right)$ (left), $S=1\left(t^{*} \approx 0.45\right)$ (center), and

$$
S=1.3\left(t^{*} \approx 0.24\right)
$$

(right. Flow conditions are summarized in Table VI

A dedicated discussion, however, is needed for the configurations with $S>1$. Interestingly, in this case the modifications are not limited to the radial contraction or expansion of the PAS (which, as explained before, is linked by a precise cause-and-effect relationship to the behavior of the underlying carrier toroidal vortex). Remarkably, changes also affect the behavior in the axial direction. As yet made evident by Fig. 11, the well-known gap of clear fluid located between the structure and the cold rod (already observed in earlier experimental investigations for liquid bridges with $S=1,[19])$ becomes almost negligible for 


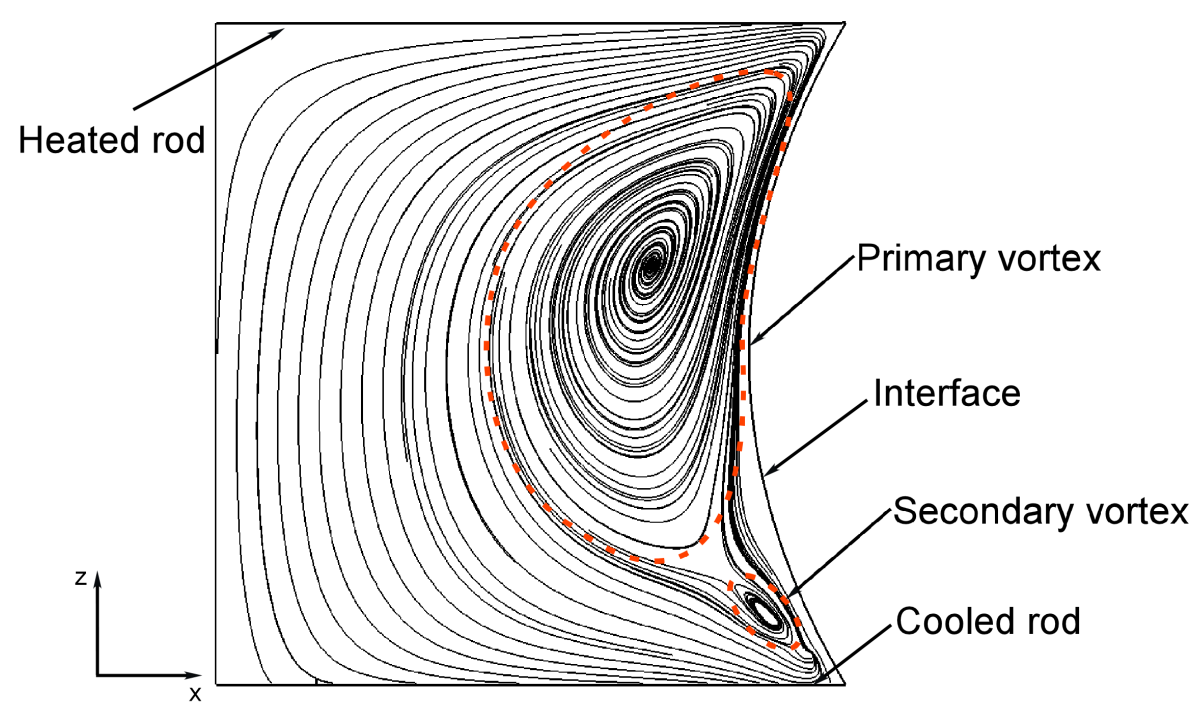

FIG. 12: Streamlines for an axis-symmetric two-dimensional simulation for $A R=0.5$ and $S=0.82$. Flow conditions are, $M a=14000$ and $P r=8$

the configurations with $S>1$. By contrast, for $S \leq 1$ the PAS is prevented from extending into the region located in proximity to the cold side (this being very evident in the lateral views reported in Fig. 11 and also appreciable in Fig. 10).

The ability of PAS to occupy the entire available axial space (grazing the cold rod) should therefore be regarded as a typical signature of particle structures emerging for $S>1$. Once again, the key to understanding these results lies in considering the behavior of the carrier flow.

On the basis of our numerical results, an explanation for this trend could be elaborated in its simplest form on the basis of the argument that the "secondary vortex" developing near the "cold corner" (cf. Fig. 12) for $S \leq 1$ is no longer present when $S$ becomes larger than 1 (as this vortex is not created in situations with larger values of the volume ratio, we infer that its presence can limit the extension of PAS in the axial direction by causing a spatial rearrangement of the structure of the KAM tori).

Interestingly, Fig. 10 and 11 also show some particles randomly distributed in the physical domain. These must obviously be regarded as an effect of the chaotic streamlines located outside the KAM torus (the reader interested in these specific dynamics being referred to the related discussions elaborated by [36]).

Towards the end to explore further the basin of attraction of the stable particle structures 
(also for $A R=0.5$ ), additional runs have been performed considering different Stokes numbers (while keeping $M a$ constant). These simulations (see Table VII) do confirm the trend already revealed by those conducted for $A R=0.34$. While for the cases with concave and straight interfaces, PAS can be obtained even for relatively small particles (some simulations were conducted considering $S t \approx 1.00 \times 10^{-8}$ ), for $S=1.3$ the minimum particle dimension has to be increased up to $S t \approx 1.30 \times 10^{-5}$.

After examining situations with $m=2$ and $m=3$, further understanding of the observed dynamics has been gained by considering situations for which the emerging flow is characterized by wavenumber $m=1(A R=0.9)$. This case is of special interest as available results are relatively rare and sparse. Indeed, if the literature extensively described in the introduction were reconsidered under a different perspective, that is, the value of the azimuthal wavenumber, it would become evident that the majority of existing studies on PAS have been conducted considering states with $m=2[5,7,10]$ or $m=3[2,6,36,37$, 42]. The amount of information about the mode $m=1$, the related multiplicity of solutions and relation with the basin of attraction is relatively limited ( [19]). What sets this specific case apart from the others discussed earlier is the very elusive nature of particle structures for this mode of convection. They seems to exist only in a very restricted range of values of the Marangoni number [19], which explains why available results are so limited.

The main outcomes of the present computations for this value of the azimuthal wavenumer have been collected in Fig. 13. Such a figure is useful as it can be used to highlight immediately the differences between the PAS for $m=1$ and those obtained for higher values of the azimuthal wavenumber. Perhaps, the most striking distinguishing mark is represented by the limited azimuthal extension of the string formed by the particles. Though it can still be interpreted as a closed circuit wrapped around the toroidal vortex, it occupies less than one quarter of the overall circumferential extension of the liquid bridge (if an axial view of the liquid bridge is considered). Another way to think about the PAS for $m=1$ is to consider an inclined single coil or closed single loop formally encapsulating a surface that intersects the axis of the liquid bridge (a similar interpretation has been provided by [19] where the PAS for $m=1$ has been described as a "deformed hook"). 

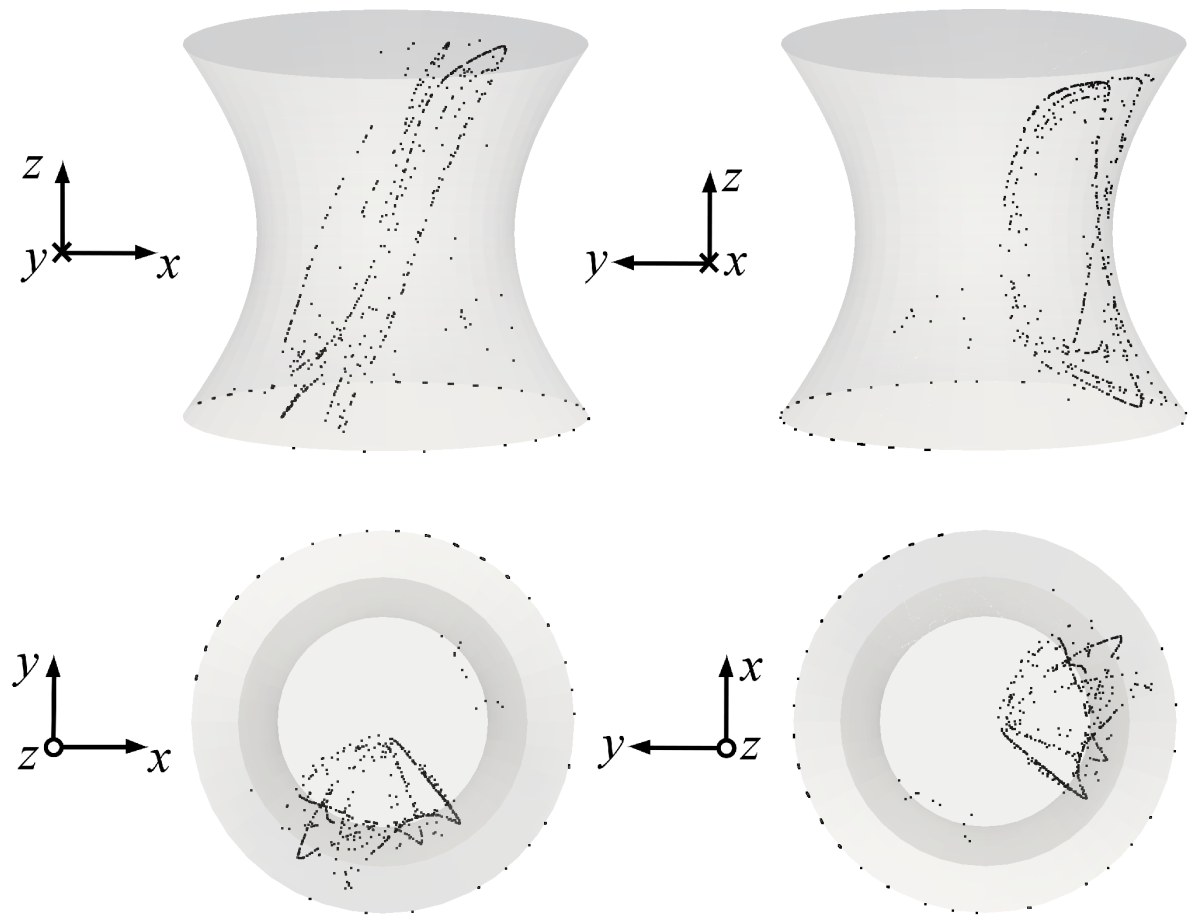

FIG. 13: Lateral (above) and axial (below) views of the PAS found for the case $A R=0.9$ and $S=0.6\left(t^{*} \approx 0.28\right)$

TABLE VII: Summary of the whole set of simulations carried out for the case $A R=0.5$ for different values of $S t$ and $\xi$. The outcome of each run is summarized in the last column

\begin{tabular}{ccccc}
\hline \hline$S$ & $M a / M a_{c}$ & $S t$ & $\xi$ & PAS \\
\hline 0.82 & 1.72 & $<1.00 \times 10^{-8}$ & 1.85 & Yes \\
1.00 & 1.68 & $<1.00 \times 10^{-8}$ & 1.85 & Yes \\
1.30 & 1.80 & $>1.30 \times 10^{-5}$ & 1.85 & Yes \\
1.30 & 1.80 & $\leq 1.30 \times 10^{-5}$ & 1.85 & No \\
\hline \hline
\end{tabular}

\section{Effect of the interface shape on PAS morphology}

The foregoing discussion has deliberately been limited to illustrating the macroscopic patterning behavior of PAS and its relationships with the azimuthal wavenumber. The present section is entirely devoted to a precise characterization of the PAS in terms of topology and (non-dimensional) extension in space as a function of the volume ratio. 


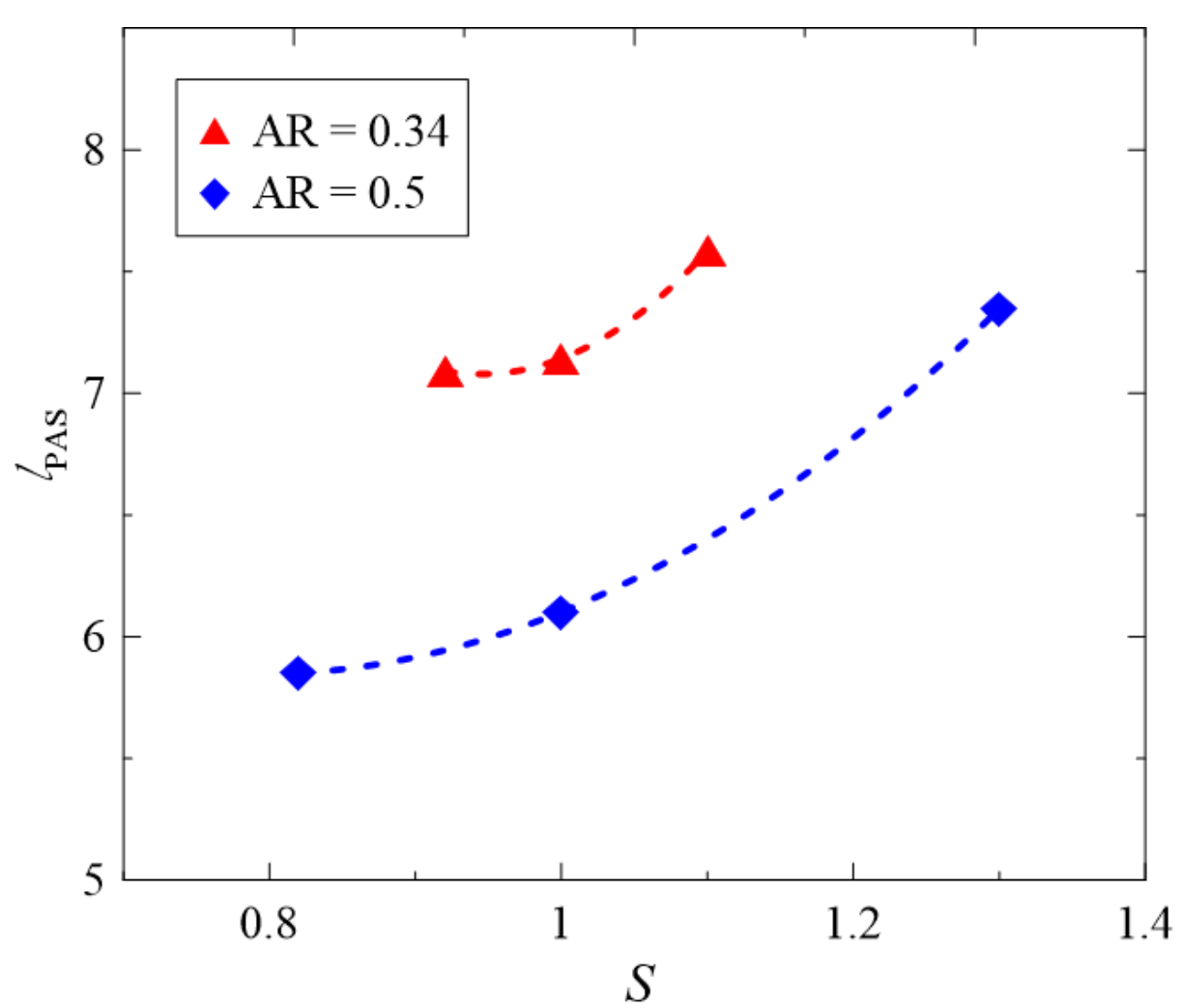

FIG. 14: Dimensionless PAS length, $l_{P A S}$, as a function of $S$ for $A R=0.34,0.5$ $\left(M a / M a_{c} \sim 2\right)$. The dashed lines represent quadratic polynomial fits

Prior to expanding on how a possible quantification of these effects can be obtained, a rigorous definition of them must be introduced. In practice, this means that the problem must be placed in a more precise theoretical context by introducing some definitions. Towards this end, we use "ad hoc" defined parameters expressly conceived to allow a quantitative characterization of these structures in a relatively straightforward way.

Though no definition is perfect, and it is hard to disentangle a definition from a property, first of all, we define the dimensionless "length" of the PAS as the linear extension of the closed circuit (i.e. the "windmill") formed by particles in space normalized with respect to the diameter of the rods, $l_{P A S}=L_{P A S} / D$.

This quantity is reported here for all the results presented in the earlier section. In order to do so, we had to calculate $L_{P A S}$ with the aid of a CAD software (by measuring precisely the length of a spline mimicking the shape of the closed loop formed by particles).

These calculations are quantitatively substantiated in Fig. 14 where the variation of the non-dimensional $l_{P A S}$ with the volume ratio is shown for the cases $A R=0.34$ and 0.5 in the 


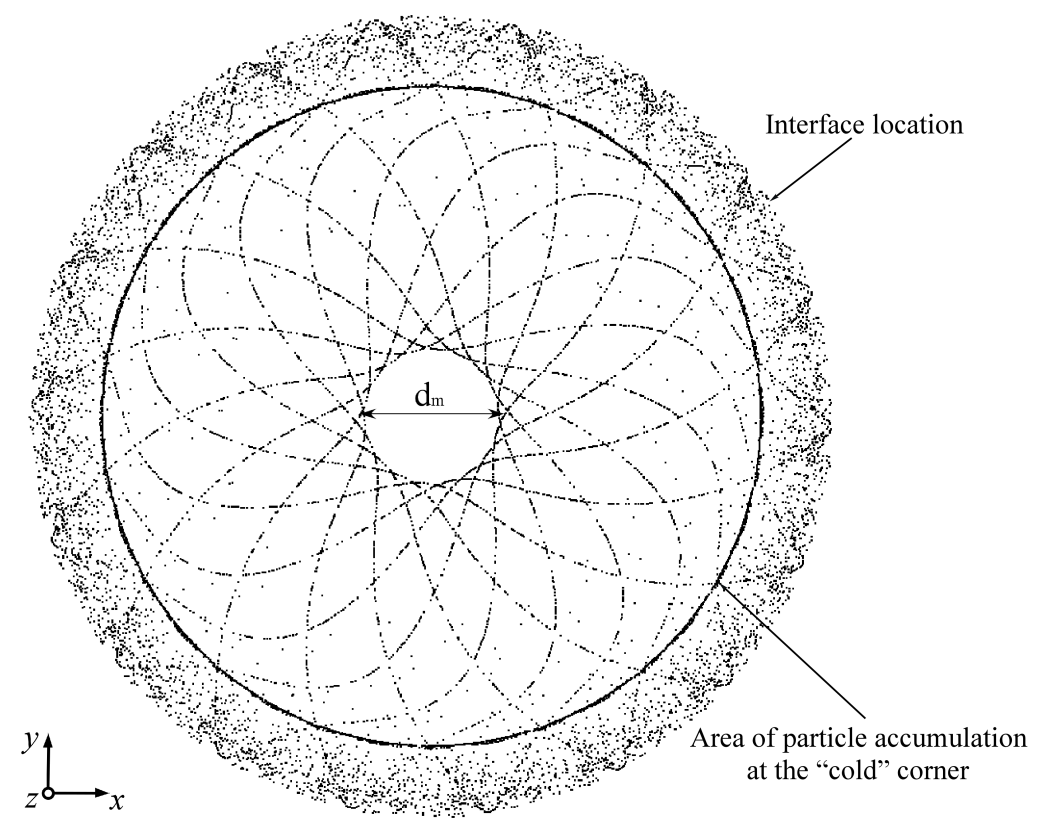

FIG. 15

Figure obtained by rotating a single time-frame (i.e., a single image of the PAS) by constant angular increments and adding it to the previous ones in the post-processing stage. The black line indicates the pinning area in correspondence of the cold disk (Conditions are: $A R=0.5$,

$$
S=0.82)
$$

form of two distinct curves.

For a proper interpretation of these results, it is convenient to start from the simple remark that the larger (non-dimensional) length of the PAS for the shallower bridge simply reflects the well-known scaling of the azimuthal wavenumber with the aspect ratio. As the aspect ratio is reduced, the azimuthal wavenumber roughly increases as $m \propto 1 / A R([27]$ and references therein). Since the number of "petals" (or blades of the windmill) is equal to the azimuthal wavenumber, when $m$ increases from 2 to 3 (as the aspect ratio is decreased from $A R=0.5$ to 0.34 ), the PAS length grows. As evident in this figure, the variation (the vertical distance separating the curves for $A=0.34$ and $A R=0.5)$ is approximately equal to one $\left(\Delta l_{P A S} \approx 1\right)$, which indirectly indicates that the length of the portion of PAS corresponding to a single "petal" or blade of the windmill can be assumed to be approximately equal to the diameter of the supporting rod.

This figure is also instrumental in showing that for each aspect ratio the length increases 


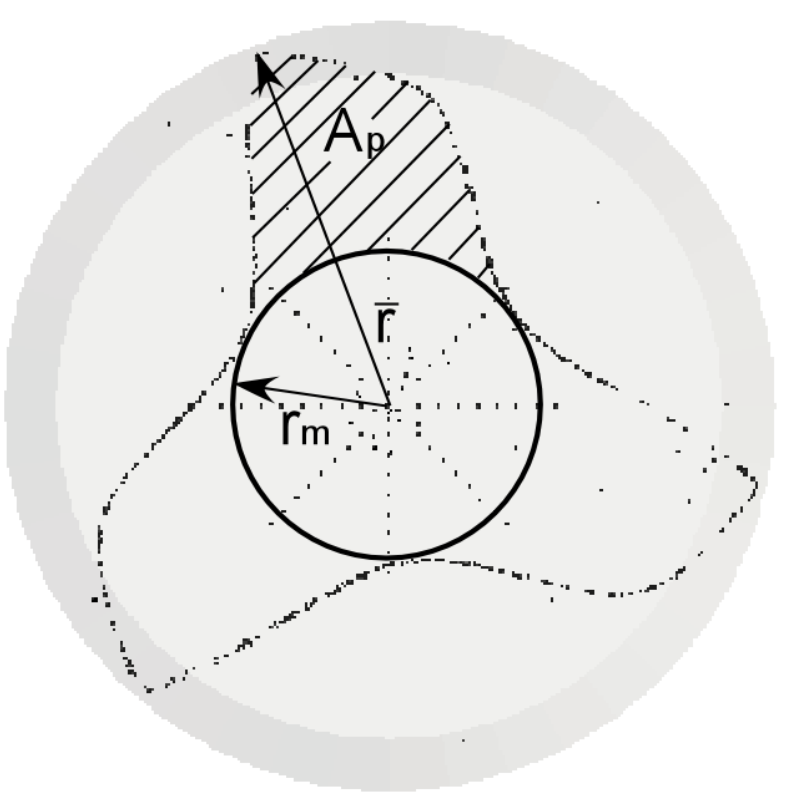

FIG. 16: Top view of the structure for the case $A R=0.34, S=1$ with a schematic depiction of the projected area of a single petal, $A_{p}$ and it maximum radial extension, $\bar{r}$. Note that $r_{m}=d_{m} / 2$ (cf. $d_{m}$ indicated in Fig. 15 )

monotonically with $S$ following a quadratic law (the reader being referred to the dashed lines). This finding is particularly interesting as it highlights the possible existence of a point of minimum in the relationship linking $l_{P A S}$ to the volume. On the basis of the common trend displayed by the curves, indeed, it is reasonable to speculate that the quantity $l_{P A S}$ would attain a minimum in the limit as the volume ratio tends to zero.

There is another interesting way to come to the same conclusion: it would be sufficient to consider that for a hypothetically infinitely thin fluid column, the PAS length would depend only on the distance between the rods, while the contribution brought to it by the radial extension would be negligible (unfortunately, these arguments cannot be further substantiated through experimental investigation since it is well known that liquid bridges cannot be indefinitely "stretched" owing to stability implications).

When the other limiting situation is considered, i.e. for increasing values of the volume ratio, as witnessed by the curves, the linear extension of the structure shows no obvious sign to reach a limit (as one would expect on the basis of geometrical considerations similar to those elaborated above for the case of very slender liquid bridges). A simple way to think 


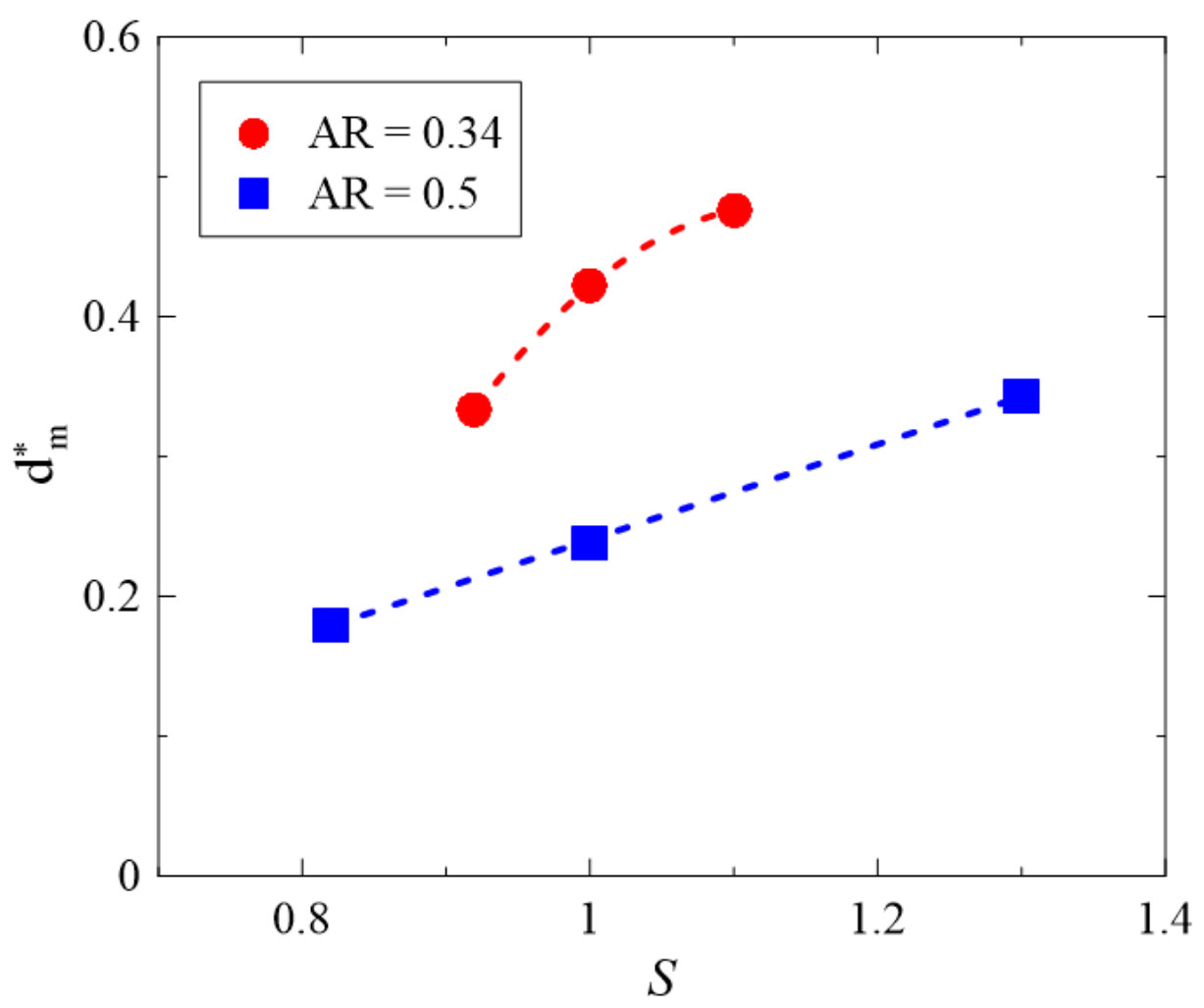

FIG. 17: Dimensionless extension of the cavity, $d_{m}^{*}$ as a function of $S$ for $A R=0.34,0.5\left(M a / M a_{c} \sim 2\right.$, the dashed lines represent quadratic polynomial fits).

about the rise of $l_{P A S}=L_{P A S} / D$ with the volume ratio is to consider that for $S \rightarrow \infty$ the PAS should possess an infinite length due to the ensuing expansion of the toroidal Marangoni roll in the radial direction. Also in this case, however, the "physics" of the considered problem, i.e. limitations stemming from stability considerations, would put a limit on the maximum volume of fluid that can effectively be held between the two rods with finite extension (diameter).

As another relevant geometrical parameter characterizing the morphology of the structure, we have used the minimum meridian extension of the surface of revolution obtained as the envelope of PAS superimposed at different time intervals (the reader being referred to Fig. 15 where this region and its diameter $d_{m}$ are reported for $A R=0.5$ and $S=0.82$ ). Notably, the same quantity was also used for the characterization of experimentally-obtained PAS by [44]. The values taken by this parameter (evaluated as $d_{m}^{*}=d_{m} / D$ ) for the present configurations are plotted in Fig. 17.

It can be seen there that also in this case data for LB with $A R=0.34$ are quantitatively 


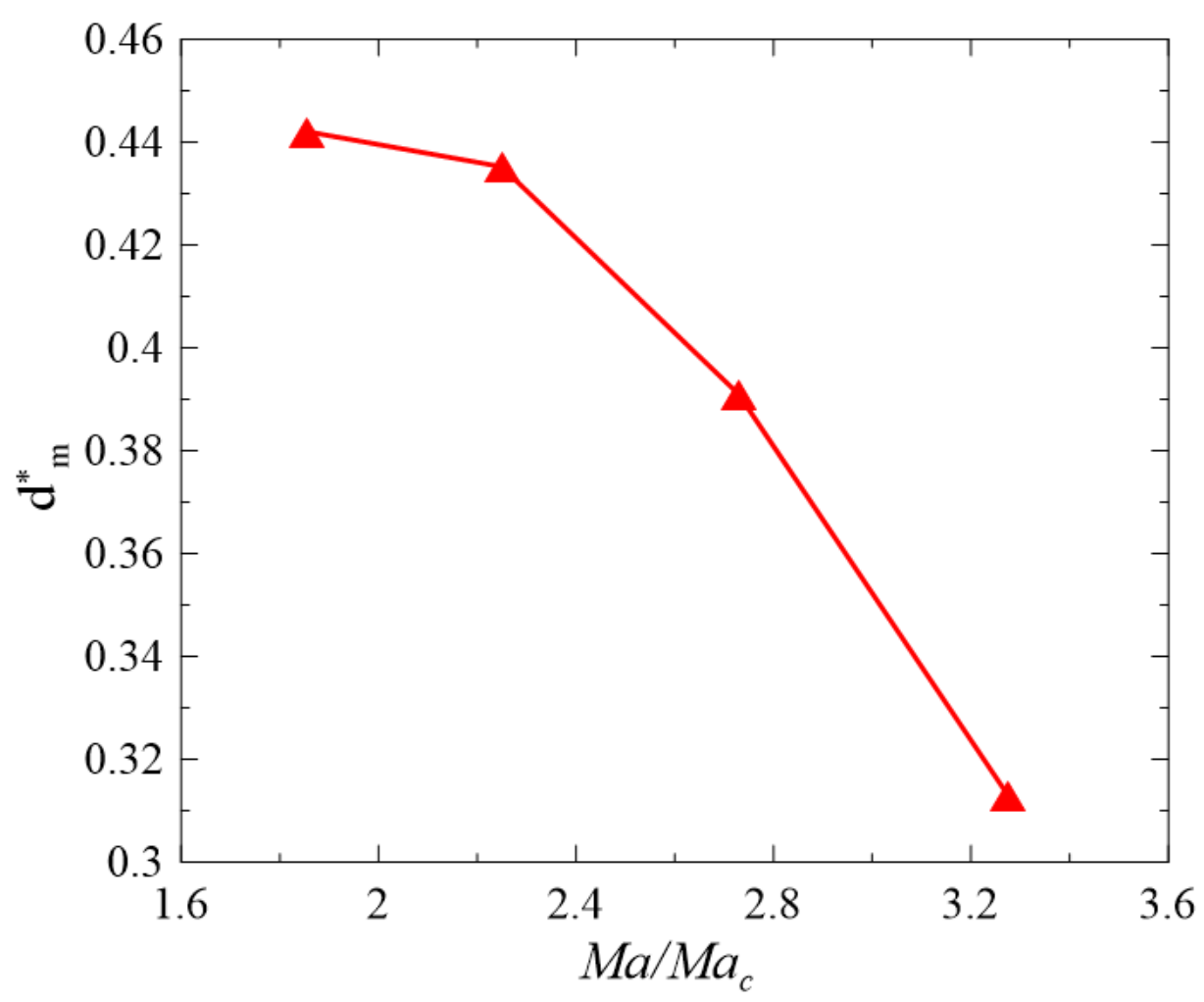

FIG. 18: Dimensionless extension of the cavity, $d_{m}^{*}$ as a function of the Marangoni number for the case $S=1$ and $A R=0.34$ ).

larger than those for the taller floating zones.

This time, the key to understanding the results lies in considering that, if the diameter of the supporting rods is kept fixed, a decrease in the height of the liquid column (i.e. in $A R$ ) will lead the toroidal vortex to be clustered progressively closer to the interface and embrace accordingly a larger percentage of quiescent fluid in the central region. In such a context, it is worth recalling that the radial depth of penetration of the toroidal roll is almost equal to the distance between the rods [66]. This implies that as the aspect ratio is decreased, the centerline of the toroidal roll must necessarily shift outwards, thereby increasing the radial extension of the central region with cold almost motionless fluid. Since PAS are known to encapsulate (or circumvent) the above-mentioned region of cold almost quiescent fluid, the above arguments may be regarded as a good rationale for the observed trend.

Other interesting information can be gathered from Fig. 18 where the dependence of $d_{m}^{*}$ on $M a$ is illustrated for $A R=0.34, S=1$ and Marangoni numbers exceeding the condition $M a / M a_{c} \sim 2$. It can be seen that this geometrical parameter decreases as a 
function of $M a$. We wish to highlight that this trend is in agreement with the earlier experimental results obtained for similar conditions $(A R=0.32, S=1)$ and different values of the Prandtl number $(\operatorname{Pr}=15$ and $\operatorname{Pr}=28.6)$ by [40] for n-decane and $2 \mathrm{c} S t$ silicone oil, respectively. They determined the minimum distance from the liquid-bridge center for the PAS $\left(R_{P A S}\right)$, using a definition that is consistent with that employed in the present work. In particular, these researchers found that $R_{P A S} \propto M a^{-\eta_{\text {pas }}}$ (cf. Fig. 7 reported in [40]) with $\eta_{\text {pas }}=1.13,1.39$ for $\operatorname{Pr}=15$ and $\operatorname{Pr}=28.6$, respectively. Notably, the present results for $M a / M a_{c}>2$ can be described using a similar law, i.e., $d_{m} \propto M a^{-\eta_{P A S}}$ with $\eta_{P A S}=0.875$ (which, among other things, further confirms the decreasing behavior of the exponent with the Prandtl number). By synergetic combination of experimental and numerical data, a possible scaling law for this exponent could therefore be expressed as $\eta_{P A S}=0.48+5.57 \times 10^{-2} \mathrm{Pr}-8.40 \times 10^{-4} \mathrm{Pr}^{2}$ (to be used as a benchmark for future experimental and/or numerical studies for intermediate values of the Prandtl number).

Continuing with the description of the results summarized in Fig. 17, it should also be stressed that for $M a / M a_{c} \sim 2$ the aspect ratio can influence sensitively the mechanism of variation of $d_{m}^{*}=d_{m} / D$ with the volume ratio $S$. Indeed, from the analysis of the two curves, fundamental differences can be noticed in the related dependencies. For the case $A R=0.34, d_{m}$ increases in a quadratic fashion (the dashed line corresponds to a polynomial interpolating expression of degree 2), while for $A R=0.5$ the diameter increases almost linearly.

Notably, an extrapolation of the linear relationship valid for $A R=0.5$ to zero $\left(d_{n}^{*} \rightarrow 0\right)$ would yield a corresponding value of the volume ratio also close to zero. Yet a reasonable interpretation for such a behavior can be elaborated in its simplest form on the basis of purely geometrical arguments. In the limit as $S$ tends to zero, the radial extension of the central region of quiescent fluid shall also tend to zero. Vice versa, on increasing $S$, the position of the toroidal roll centerline will be displaced outwards, thereby causing an expansion of that area.

A more involved explanation, however, is required when the other aspect ratio $(A R=$ 0.34) is considered. Related data seem to indicate that $d_{m}$ cannot grow indefinitely as $S \rightarrow \infty$. The different (quadratic) scaling law also provides important clues in this regard. These data suggest the existence of a maximum allowable radial extension of the inner diameter of the PAS in the considered range of values of the volume ratio. 


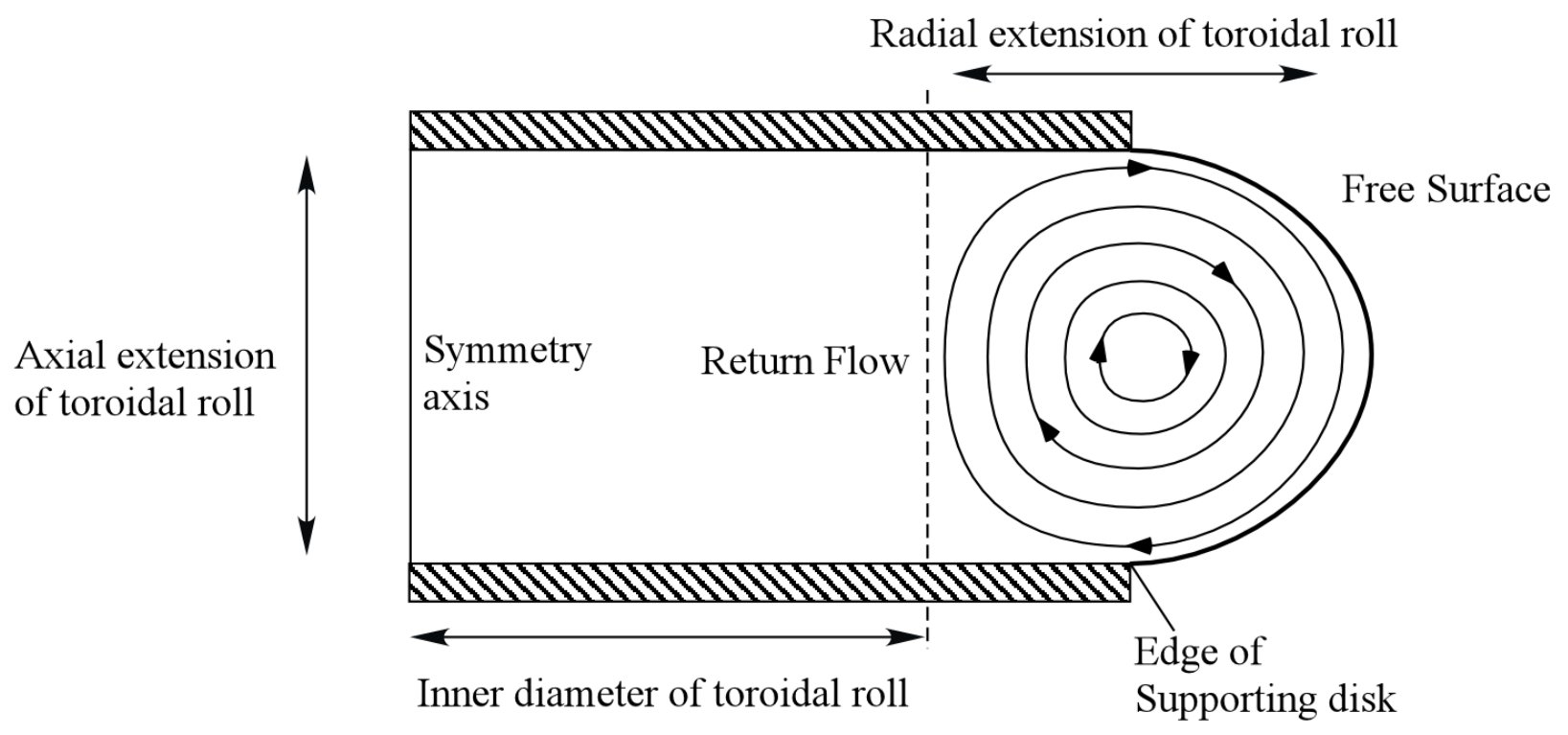

FIG. 19: Location of the main toroidal vortex in a shallow liquid bridge with convex interface - the deformation of the free surface has been exaggerated for illustration purposes.

In the light of the arguments elaborated above, this asymptotic condition may be considered equivalent to the existence of a maximum radial extension of the inner region of quiescent fluid.

Using this perspective, a heuristic interpretation for the presence of a limit in the curve for $A R=0.34$ could be elaborated as follows: while for larger values of the aspect ratio $(A R=0.5)$, an increase in $S$ can cause a significant expansion of the inner quiescent core (with respect to the diameter of the supporting rod) due to the significant radial displacement undergone by the toroidal roll centerline, the corresponding (same value of $S$ ) relative displacement for shallower liquid bridges will (obviously) be more contained. In practice, another factor contributing to put a limit on the maximum attainable value of $d_{m}$ for liquid bridges with small aspect ratio is represented by the constraint that, in any case, the toroidal roll will tend to retain an inner boundary located at a radial distance from the axis of the liquid bridge that is smaller than the radius of the supporting disks. Put simply, this "inner boundary" corresponds to the return flow that is created due to continuity effects in the cylindrical sub-region of fluid delimited from above and below by the two supporting disks (see Fig. 19)

The most interesting outcome of these observations, perhaps is that, while $l_{P A S}=$ 


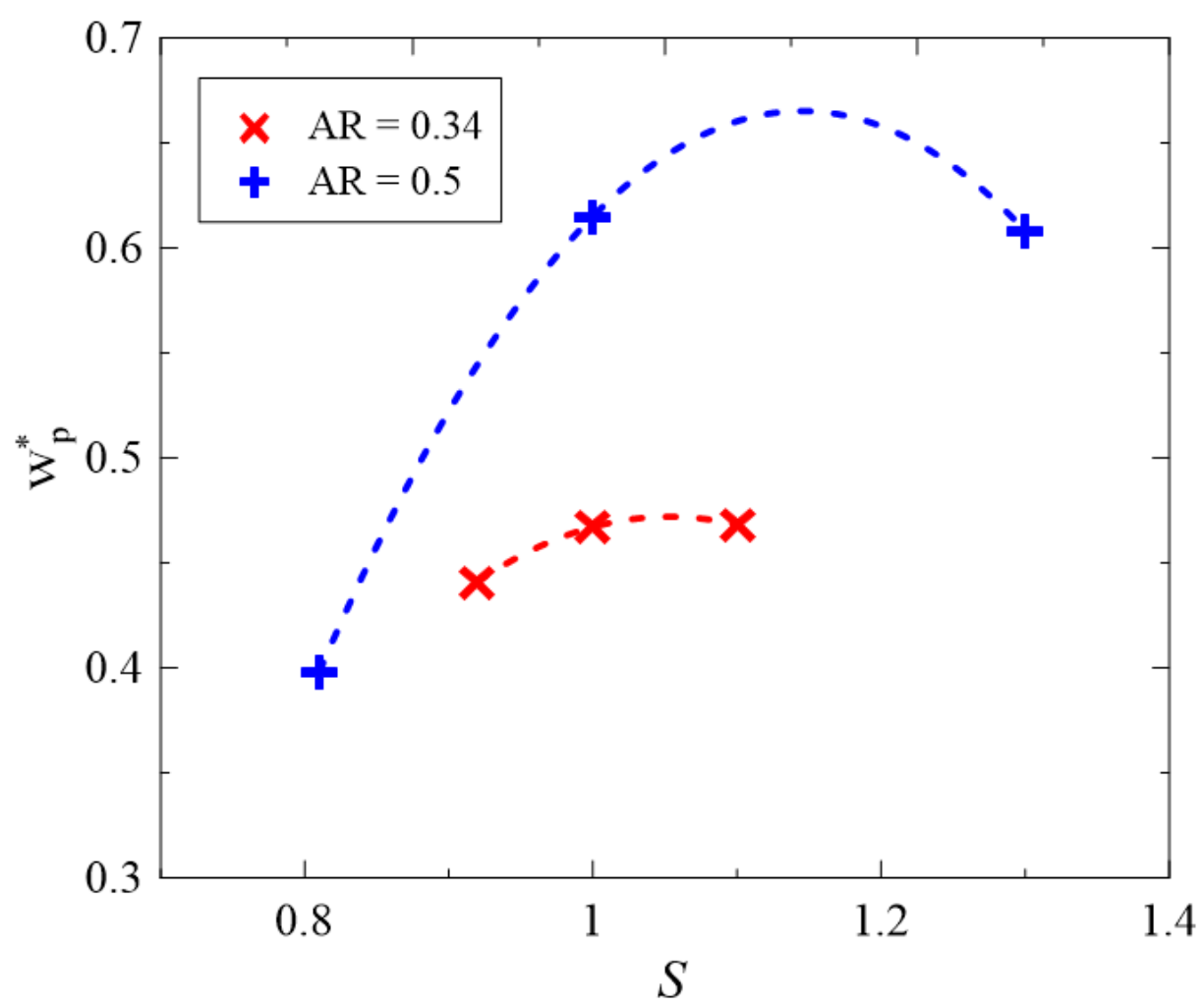

FIG. 20: Dimensionless width of a single PAS petal as a function of the volume ratio $\left(M a / M a_{c} \sim 2\right)$. The dashed lines represent quadratic polynomial fits

$L_{P A S} / D$ would grow indefinitely with the volume ratio (if this ratio could be made infinite), similar considerations do not apply to $d_{m}$.

There is also another interesting way to use all these considerations to justify the absence of a visible asymptotic behavior in the trend of $d_{m}$ for $A R=0.5$. For this specific aspect ratio (and larger values of $A R$ ) the internal region of quiescent fluid will obviously be less extended (with respect to the diameter of the supporting rod). This means that values of the volume ratio larger than those for which the limit condition becomes effective for $A R=0.5$ would be needed. We argue that for $A R=0.5$ or larger $A R$ the asymptotic condition would be attained for a value of $S$ located outside the interval of values examined in the present work.

The remainder of this section is finally devoted to discuss the scaling behavior of another parameter specifically introduced as a fruitful alternative for obtaining additional insights into the morphology and topology of all these structures, that is, $w_{p}^{*}$, namely the "mean" extension of a single PAS petal in the azimuthal direction. 
A similar parameter was previously used by [8]. In the present work, however, we make its definition more general and independent from the radial coordinate. Towards this end, (see Fig. 16), in particular, first we define the area of a single petal, $A_{p}$, as the area delimited (externally) by the projection of the three-dimensional PAS line on a plane perpendicular to the bridge axis and (internally) by the arc of circumference corresponding to the inner diameter $d_{m}$. Then, we determine accordingly the mean azimuthal extension of the petal as the ratio of such area and its radial extension, i.e., $w_{p}=A_{p} /\left(\bar{r}-r_{m}\right)$. Finally, this quantity is made nondimensional using the radius of the rods as reference length, i.e., $w_{p}^{*}=2 w_{p} / D$.

Along these lines, Figure 20 shows the plots $w_{p}^{*}$ vs $S$ again for the two aspect ratios examined before. In this case we have found the data to be well fitted by parabolic curves with concavity facing downward. Remarkably, this implies that for each curve it is possible to identify a well-defined point of maximum separating the curve into branches with opposite behaviors (namely, parts of the curve where the dimensionless width becomes increasingly smaller if the volume ratio, $S$ is increased or decreased, respectively). Interestingly, the maximum mean width (or area of the petal) is attained in both cases for a volume ratio in the range between $S=1$ and $S=1.2$. We argue that an explanation for this nonmonotone behaviour should be sought in the interplay between two counteracting effects, namely, the tendency of the width of the petal measured at a fixed distance from the interface (approximately $20 \%$ of the rod diameter) to become smaller as the volume ratio is increased (as originally observed experimentally by [8] in normal gravity and also confirmed by the present results in microgravity conditions, see Figs. 10 and 11) and the opposite tendency of $d_{m}$ (which by growing causes an increase in the area of the petal).

Before moving to the next section, we wish also to highlight that the evident difference affecting the two curves in terms of magnitude (average value) simply indicates that the mean (non-dimensional) azimuthal extension of the petals becomes smaller as the wavenumber increases (compare the morphology of the petals in Figs 10 and 11). The different 'sensitivity' to $S$ (it being much more evident for $A R=0.5$ ) can be explained taking into account the different relative deformation undergone by the interface for a fixed volume ratio and different values of the aspect ratio. For a fixed $S$, the relative deformation will obviously be higher for larger values of $A R$. 

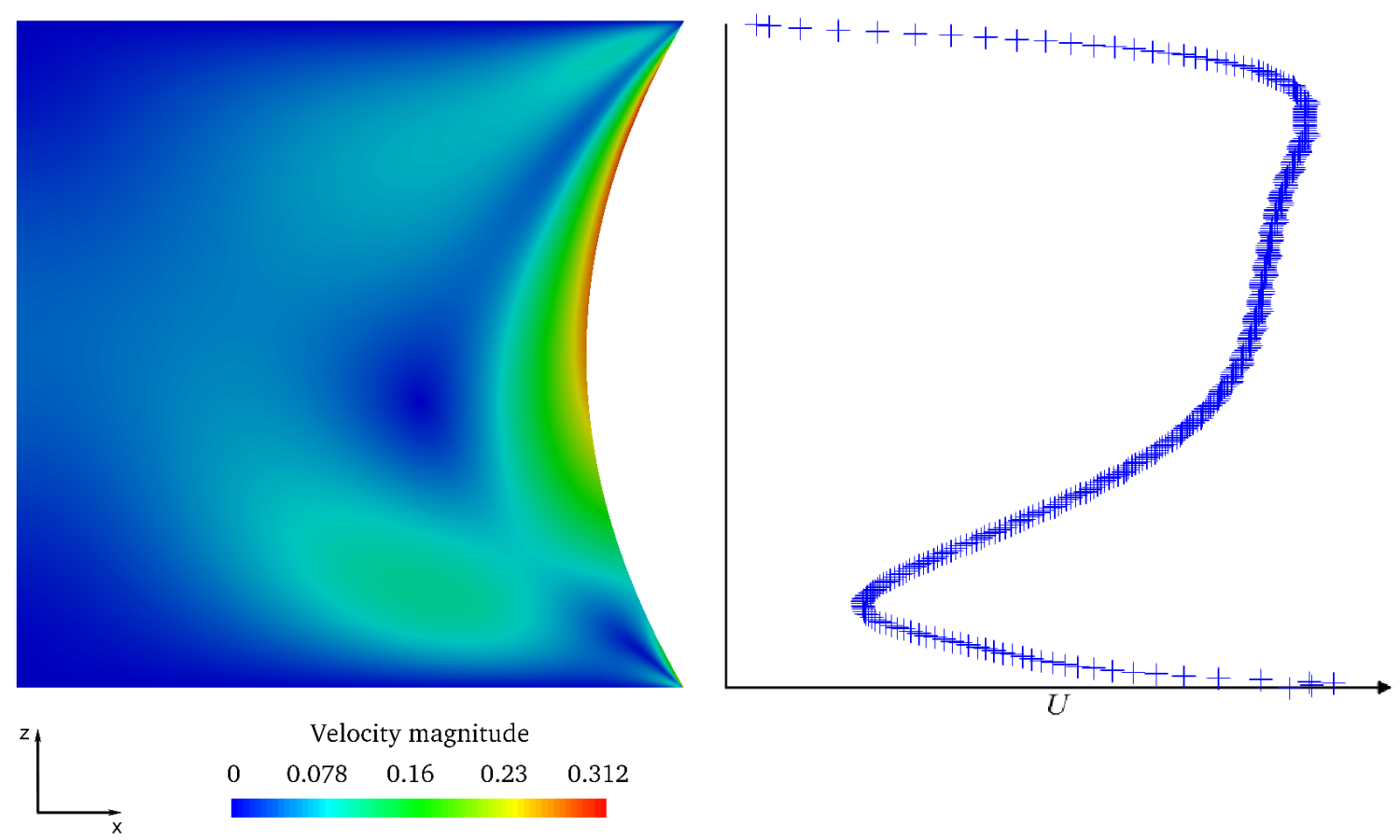

FIG. 21: Module of the dimensionless velocity field (left) and velocity distribution on its interface (right) for a two-dimensional, concave LB. The flow conditions adopted were

$$
\operatorname{Pr}=8, A R=0.5, S=0.82 \text { and } M a=18000
$$

\section{Influence of interface curvature on PAS formation}

Having completed a thorough analysis of PAS topological and morphological details, this final section is devoted to a critical discussion of the possible cause-and-effect relationships at the root of the tendency of slender liquid bridges $(S<1)$ to support the emergence of these structures over an increased range of values of the particle Stokes number with respect to cases with convex interfaces. In the present section, we attempt to provide a relatively simple interpretation of this trend on the basis of kinematic and dynamic considerations and earlier models yielded by different investigators (the reader interested in these models being referred to the literature extensively cited in the introduction).

To elaborate relevant arguments, in particular, we start from relatively simple observations, that is, the behavior of the fluid velocity along the free interface (dimensionless velocity $\left(U^{*}=U / U_{\text {ref }}\right.$, where $\left.U_{\text {ref }}=\nu / H\right)$ profile being shown in Fig. 21 for a representative case). This velocity attains a maximum in proximity to the hot wall and then decreases 

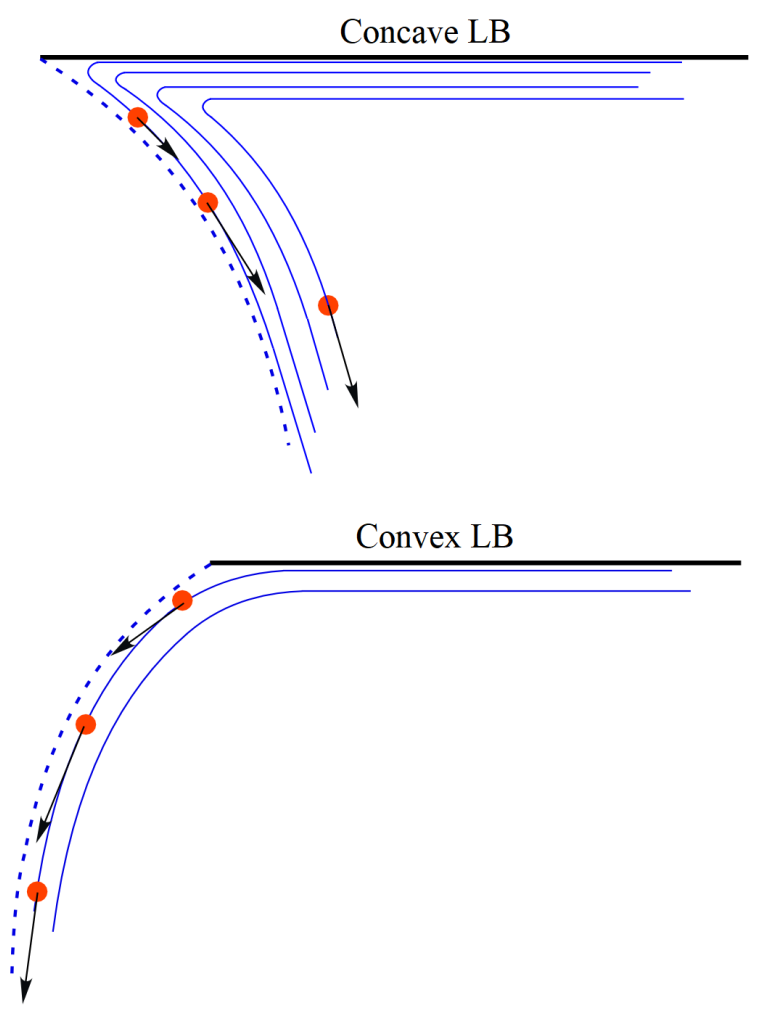

FIG. 22: Schematics of the trajectories described by a particle next to the interface moving toward the "cold" corner of the LB for two opposite configurations: concave interface (top), and convex (below). The dashed line indicates the free surface.

continuously along the interface until it starts to rise again near the cold corner (due to the presence of a thermal boundary layer adjacent to the cold side). As evident in Fig. 21 , in particular, the portion of the profile where velocity decreases is relatively extended in the axial direction (corresponding to more than half of the length of the liquid bridges, regardless of the considered specific volume ratio).

The key to elaborating the aforementioned interpretation relates to the sensitivity of non-isodense particles to velocity changes. PAS such as those considered here are known to be produced by small trajectory "defects", i.e. departure of the particle trajectory from the underlying fluid streamlines due to a mismatch in the particle density with respect to that of the host fluid. These minute defects can accumulate in time leading particles to migrate from their original streamlines to different regions of the fluid domain.

For $\xi>1$, being denser than the carrier fluid, particles moving in proximity to the free surface in the region where the surface velocity decreases in the flow direction will be 


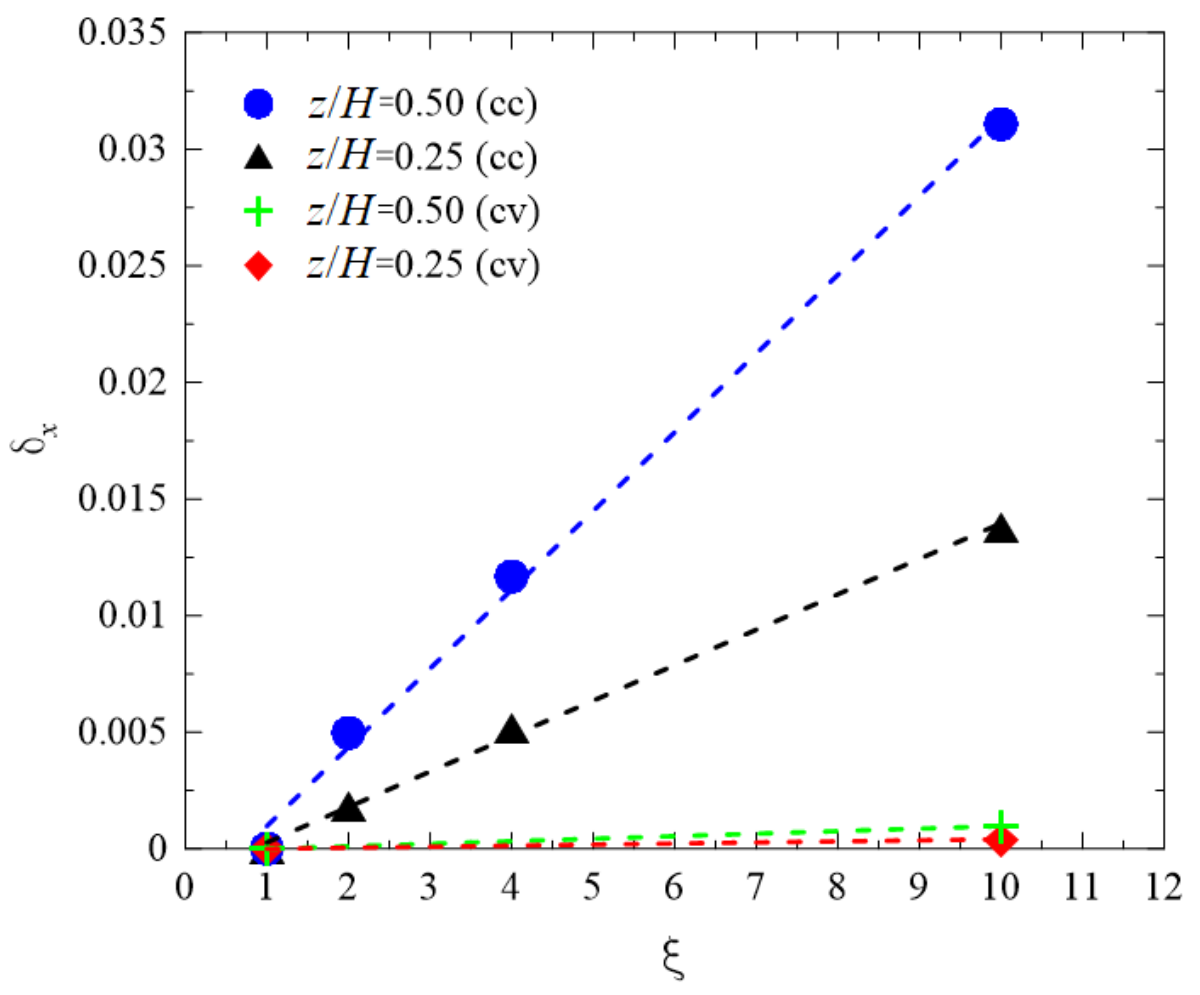

FIG. 23: Deviation of the trajectory of a single particle (evaluated with respect to the reference position obtained for the case $\xi=1$ ) as a function of the density ratio for two different LB configurations: concave bridge $A R=0.5$ and $S=0.82$ (cc), and $A R=0.5$ and $S=1.3(\mathrm{cv})$. The positions are taken in correspondence of two different cross sections

$$
\text { at } z / L=1 / 2 \text { and } z / L=1 / 4 \text {. }
$$

subjected to a force having the same direction of the fluid velocity. To understand how this works from a physical point of view it is sufficient to consider that when the fluid decelerates, owing to the density difference, the velocity of particles will tend to exceed that of the surrounding liquid.

This observation becomes particularly useful if it is considered together with the curvature of the free interface for $S<1$ and $S>1$.

As these two situations are one the mirror image of the other, the component of the abovementioned force in the radial direction will take opposite sign for the two configurations. More specifically, it will be directed inward for the concave shape and outward for the opposite condition in which the interface is convex. Coupling of merely geometrical and dynamics conditions will therefore lead to a diametrically opposite effect in terms of radial displacement of the generic particle. While for $S<1$ particles will be forced to leave their 
original streamline in favor of a more internal streamline, for $S>1$ they will tend to be displaced towards the interface and accumulate there, accordingly.

The evolution (in terms of trajectory) of a particle next to the heated rod that travels first toward the corner region and then along the interface from the hot side towards the cold one is qualitatively sketched in Fig. 22 (the particle position is reported there at different times for a liquid bridge with a concave (top view), and convex (bottom view) free-surface, respectively).

For $S<1$, as soon as the particle enters the above-mentioned region where surface fluid velocity decreases, even minutes departure of the particle from its original trajectory due to its different density will cause transfer of the particle to another streamline (thereby contributing to the mechanism by which Lagrangian coherent structures can be formed [3, 5, 10, 30-32]. Vice versa, for $S>1$, the density jump would act just in the opposite way, tending to push particles towards the free interface.

These qualitative arguments are quantitatively substantiated by the data reported in Fig. 23 where we have summarized the results provided by high-resolution two-dimensional (axi-symmetric) simulations (grid $360 \times 360$ ). This figure shows the radial displacement undergone by particles that are initially located near the hot rod as a function of the density ratio and two values of the volume ratio for $A R=0.5$. In order to make the variations evident, in particular, the "differential" radial displacement, $\delta_{x}$, has been plotted, that is, the difference between the radial position of the considered particle for a given value of the density ratio $(\xi)$ and the radial position (at the same axial location) that would be taken by a particle with the same density of the surrounding fluid $(\xi=1)$.

As witnessed by this figure, the particle differential radial displacement relating to the liquid bridge with $S>1$ is negligible with respect to the equivalent values obtained for the same density ratios and $S<1$. This supports the above explanation elaborated taking into account the different role played by the relative density of particles in relation to the different (concave or convex) shape of the liquid bridge.

At this stage, we wish to emphasize that, in order to filter out the role of numerical error as a possible source of "unphysical" noise (mimicking the effects of a density mismatch and potentially leading to "artifacts" for $S<1)$, we have conducted additional numerical (3-D) simulations for the case $A R=0.5$ and $S=0.82$ and for the smallest Stokes number shown in Table VII using a different computational platform (Ansys Fluent). The same simulation 
has been repeated using a 4th order accurate Runge-Kutta method in combination with the highest mesh resolution $M_{3}$ (cf. III). no changes have been found in the emerging PAS.

As a concluding remark for this section, we wish also to provide some interesting reflections about the 3 -D cases with $S>1$. Owing to the effect elucidated above, by which particles with different density ratios $(\xi>1)$ moving in proximity to the free interface are forced to follow the same trajectory as their density were almost equal to that of the surrounding fluid, it is reasonable to assume that the alternate formation mechanism proposed by Kuhlmann and co-workers may play a substantial role in this case. This model ([34-36], based on the paradigm of 'finite-size' Lagrangian coherent structures, would indeed be operative in the regions along the free interface where velocity increases along the flow direction (that is, in proximity to the hot and cold rods). Future work shall be devoted to a precise assessment of this effect for liquid bridges with $S>1$ by introducing in the boundary interaction model the so-called "lubrication thickness" [36] and evaluating its impact on the ranges of particles Stokes number for which PAS can effectively be obtained (including a precise determination of the related formation time).

\section{CONCLUSIONS AND FUTURE WORK}

The recent resurgence in interest in the so-called PAS has led to significant advances in our understanding of the origin and properties of these phenomena, as witnessed by the various models proposed by different research groups to explain the underlying mechanisms. As a critical analysis of the existing literature would reveal, however, most of the past theoretical and numerical research into this subject has been overwhelmingly devoted to the case of liquid bridges with straight surface (perfectly cylindrical shape). The present work has continued these investigations by probing the role of a parameter almost ignored in the literature, that is the effective volume of liquid held between the supporting rods.

Building on, but also seeking to extend, the valuable research that has highlighted the role of the density difference and flow topology in supporting the emergence of these particle structures, we have explored a relatively wide portion of the space of parameters considering liquid bridges with different aspect ratios and volume ratios and conditions for which particle inertia is expected to be significant. In particular, insights have been sought from focused comparisons of two archetypal settings, namely, liquid bridges with concave and 
convex free surfaces in microgravity conditions.

In a broad outline, our findings have revealed that the morphology of the emerging structures is largely affected by the deformation of the interface. Geometrical parameters have been expressly defined with the intent to quantify precisely the morphological variations undergone by these structures as a result of liquid volume (and aspect ratio) changes.

Whenever possible, scaling laws for these parameters have been identified and an interpretation of the related trends has been provided.

Interestingly, while the non-dimensional linear extension ("length") of the PAS shows no obvious limit when the volume of fluid is increased, as asymptotic behavior can be clearly identified for its "inner diameter" if relatively small values of the aspect ratio are considered. In terms of axial extension, PAS grow when the liquid volume is increased. Following the non-trivial interplay among counteracting effects, however, a non-monotone law of variation is obtained when a characterization of the PAS morphology is attempted in terms of the area of its projection in planes perpendicular to the axis of the liquid bridge (the windmill "as seen from above").

Though the main aim of the present study was to provide a set of predictive links between geometry, flow properties and particle behavior, some problems have also been used as an opportunity for discussing fundamental issues that are shared among the two main existing (concurrent) theories for the explanation of PAS, and putting them in relation with the specific dynamics considered here.

We have found that particle density effects can play a diametrically opposite role if they are considered in conjunction with concave or convex liquid bridges respectively. Owing to the existence of an extended region along the free surface of the liquid bridge where the surface flow is decelerated, particles denser than the carrier fluid that move in proximity to the interface will experience a force with radial component directed inward or outward depending on whether the volume of liquid is smaller or larger than the equivalent cylindrical one. An explanation for the increased ability (as provided by the numerical simulations) of concave liquid to support the emergence of PAS with respect to convex ones can therefore be directly rooted in the interplay between purely topological flow properties and the forces acting on the particles mainly as a result of their different density with respect to the fluid. Future work shall be devoted to assessing precisely the role of particles finite-size effects in the regions where the surface fluid is accelerated (e.g., in proximity to the hot corner) and 
their ability to support the emergence of PAS in convex liquid bridges.

\section{ACKNOWLEDGMENTS}

This work has been supported by the UK Engineering and Physical Sciences Research Council (EPSRC grant EP/R043167/1) and the UK Space Agency in the framework of the JEREMI (Japanese European Research Experiments on Marangoni Instabilities) ESAJAXA project.

\section{REFERENCES}

${ }^{1}$ M. Lappa, "On the formation and morphology of coherent particulate structures in nonisothermal enclosures subjected to rotating g-jitters", Physics of Fluids 31, 073303 (2019).

${ }^{2}$ D. Schwabe and A. I. Mizev, "Particles of different density in thermocapillary liquid bridges under the action of travelling and standing hydrothermal waves", European Physical Journal Special Topics 192, 13-27 (2011).

${ }^{3}$ D. O. Pushkin, D. E. Melnikov, and V. M. Shevtsova, "Ordering of small particles in onedimensional coherent structures by time-periodic flows", Phys. Rev. Lett. 106, 234501 (2011).

${ }^{4}$ D. Melnikov, D. Pushkin, and V. Shevtsova, "Accumulation of particles in time-dependent thermocapillary flow in a liquid bridge. modeling of experiments", The European Physical Journal Special Topics 192, 29-39 (2011).

${ }^{5}$ D. E. Melnikov, D. O. Pushkin, and V. M. Shevtsova, "Synchronization of finite-size particles by a traveling wave in a cylindrical flow", Physics of Fluids 25, 092108 (2013).

${ }^{6}$ I. Ueno, Y. Abe, K. Noguchi, and H. Kawamura, "Dynamic particle accumulation structure (pas) in half-zone liquid bridge - reconstruction of particle motion by 3-d ptv", Advances in Space Research 41, 2145-2149 (2008).

${ }^{7}$ M. Gotoda, D. E. Melnikov, I. Ueno, and V. Shevtsova, "Experimental study on dynamics of coherent structures formed by inertial solid particles in three-dimensional periodic flows", Chaos: An Interdisciplinary Journal of Nonlinear Science 26, 073106 (2016). 
${ }^{8}$ Y. Abe, I. Ueno, and H. Kawamura, "Effect of shape of hz liquid bridge on particle accumulation structure (pas)", Microgravity Science and Technology 19, 84-86 (2007).

${ }^{9}$ M. Gotoda, T. Sano, T. Kaneko, and I. Ueno, "Evaluation of existence region and formation time of particle accumulation structure (pas) in half-zone liquid bridge", The European Physical Journal Special Topics 224, 299-307 (2015).

${ }^{10}$ D. E. Melnikov and V. Shevtsova, "Different types of lagrangian coherent structures formed by solid particles in three-dimensional time-periodic flows", The European Physical Journal Special Topics 226, 1239-1251 (2017).

${ }^{11}$ F. Balboa Usabiaga and R. Delgado-Buscalioni, Particle hydrodynamics: from molecular to colloidal fluids (Springer Nature, 2011), pp. 152-163.

${ }^{12}$ E. Saeedi, S. Abbasi, K. F. B. ohringer, and B. A. Parviz, "Molten-alloy driven self-assembly for nano and micro scale system integration", Fluid Dynamics \& Materials Processing 2, 221-246 (2006).

${ }^{13}$ M. Ohta and M. Sussman, "The buoyancy-driven motion of a single skirted bubble or drop rising through a viscous liquid", Physics of Fluids 24, 112101 (2012).

${ }^{14}$ J. Z. Zhao, L. Ratke, and B. Feuerbacher, "Microstructure evolution of immiscible alloys during cooling through the miscibility gap", Modelling and Simulation in Materials Science and Engineering 6, 123-139 (1998).

${ }^{15}$ J. Eaton and J. Fessler, "Preferential concentration of particles by turbulence", International Journal of Multiphase Flow 20, 169-209 (1994).

${ }^{16}$ Z. Saghir and A. Mohamad, "Effectiveness in incorporating brownian and thermophoresis effects in modelling convective flow of water-al2o3 nanoparticles", International Journal of Numerical Methods for Heat Fluid Flow 28, 00-00 (2017).

${ }^{17} \mathrm{M}$. Lappa, "On the transport, segregation, and dispersion of heavy and light particles interacting with rising thermal plumes", Physics of Fluids 30, 033302 (2018).

${ }^{18}$ S. Tanaka, H. Kawamura, I. Ueno, and D. Schwabe, "Flow structure and dynamic particle accumulation in thermocapillary convection in a liquid bridge", Physics of Fluids 18, 067103 (2006). 
${ }^{19}$ D. Schwabe, A. I. Mizev, M. Udhayasankar, and S. Tanaka, "Formation of dynamic particle accumulation structures in oscillatory thermocapillary flow in liquid bridges", Physics of Fluids 19, $072102(2007)$.

${ }^{20}$ V. Shevtsova, A. Mialdun, H. Kawamura, I. Ueno, K. Nishino, and M. Lappa, "Onset of hydrothermal instability in liquid bridge. experimental benchmark", Fluid Dynamics \& Materials Processing 7, 1-28 (2011).

${ }^{21}$ S. Frank and D. Schwabe, "Temporal and spatial elements of thermocapillary convection in floating zones", Experiments in Fluids 23, 234-251 (1997).

${ }^{22}$ I. Ueno, S. Tanaka, and H. Kawamura, "Oscillatory and chaotic thermocapillary convection in a half-zone liquid bridge", Physics of Fluids 15, 408-416 (2003).

${ }^{23}$ D. E. Melnikov, V. M. Shevtsova, and J. C. Legros, "Onset of temporal aperiodicity in high prandtl number liquid bridge under terrestrial conditions", Physics of Fluids 16, 1746-1757 (2004).

${ }^{24}$ D. Melnikov, V. Shevtsova, and J. Legros, "Route to aperiodicity followed by high prandtl-number liquid bridge. 1-g case", Acta Astronautica 56, 601-611 (2005).

${ }^{25}$ K. Nishino, T. Yano, H. Kawamura, S. Matsumoto, I. Ueno, and M. K. Ermakov, "Instability of thermocapillary convection in long liquid bridges of high prandtl number fluids in microgravity", Journal of Crystal Growth 420, 57-63 (2015).

${ }^{26}$ Q. Kang, D. Wu, L. Duan, L. Hu, J. Wang, P. Zhang, and W. Hu, "The effects of geometry and heating rate on thermocapillary convection in the liquid bridge", Journal of Fluid Mechanics 881, 951-982 (2019).

${ }^{27}$ M. Lappa, Thermal convection: patterns, evolution and stability (John Wiley and Sons, Ltd, 2009).

${ }^{28}$ D. Schwabe, P. Hintz, and S. Frank, "New features of thermocapillary convection in floating zones revealed by tracer particle accumulation structures (pas)", Microgravity Science and Technology 9, 163-168 (1996).

${ }^{29}$ H. C. Kuhlmann, M. Lappa, D. Melnikov, R. Mukin, F. H. Muldoon, D. Pushkin, V. Shevtsova, and I. Ueno, "The jeremi-project on thermocapillary convection in liquid bridges. part a: overview of particle accumulation structures", Fluid Dynamics \& Materials Processing 10, 1-10 (2014). 
${ }^{30} \mathrm{M}$. Lappa, "On the nature, formation and diversity of particulate coherent structures in microgravity conditions and their relevance to materials science and problems of astrophysical interest", Geophysical \& Astrophysical Fluid Dynamics 110, 348-386 (2016).

${ }^{31}$ M. Lappa, "Assessment of the role of axial vorticity in the formation of particle accumulation structures in supercritical marangoni and hybrid thermocapillary-rotation-driven flows", Physics of Fluids 25, 012101 (2013).

${ }^{32} \mathrm{M}$. Lappa, "On the existence and multiplicity of one-dimensional solid particle attractors in timedependent rayleigh-bénard convection", Chaos: An Interdisciplinary Journal of Nonlinear Science 23, 013105 (2013).

${ }^{33}$ M. Lappa, "Stationary solid particle attractors in standing waves", Physics of Fluids 26, 013305 (2014).

${ }^{34}$ R. V. Mukin and H. C. Kuhlmann, "Topology of hydrothermal waves in liquid bridges and dissipative structures of transported particles", Phys. Rev. E 88, 053016 (2013).

${ }^{35}$ F. H. Muldoon and H. C. Kuhlmann, "Coherent particulate structures by boundary interaction of small particles in confined periodic flows", Physica D: Nonlinear Phenomena 253, 40-65 (2013).

${ }^{36}$ F. Romanò and H. C. Kuhlmann, "Finite-size lagrangian coherent structures in thermocapillary liquid bridges", Phys. Rev. Fluids 3, 094302 (2018).

${ }^{37}$ A. Toyama, M. Gotoda, T. Kaneko, and I. Ueno, "Existence conditions and formation process of second type of spiral loop particle accumulation structure (sl-2 pas) in half-zone liquid bridge", Microgravity Science and Technology 29, 263-274 (2017).

${ }^{38}$ Y. Niigaki and I. Ueno, "Formation of particle accumulation structure (pas) in half-zone liquid bridge under an effect of thermo-fluid flow of ambient gas", Transaction of the Japan Society for Aeronautical and Space Sciences, Aaerospace Technology Japan 10, 33-37 (2012).

${ }^{39} \mathrm{~K}$. Yamaguchi, T. Hori, and I. Ueno, "Long-term behaviors of a single particle forming a coherent structure in thermocapillary- driven convection in half-zone liquid bridge of high prandtl-number fluid", Int. J. Microgravity Sci. Appl. (2019).

${ }^{40}$ M. Gotoda, A. Toyama, M. Ishimura, T. Sano, M. Suzuki, T. Kaneko, and I. Ueno, "Experimental study of coherent structures of finite-size particles in thermocapillary liquid bridges", Phys. Rev. Fluids 4, 094301 (2019). 
${ }^{41}$ T. Oba, A. Toyama, T. Hori, and I. Ueno, "Experimental study on behaviors of low-stokes number particles in weakly chaotic structures induced by thermocapillary effect within a closed system with a free surface", Phys. Rev. Fluids 4, 104002 (2019).

${ }^{42}$ M. Lappa, "On the variety of particle accumulation structures under the effect of g-jitters", Journal of Fluid Mechanics 726, 160-195 (2013).

${ }^{43}$ H. C. Kuhlmann and F. H. Muldoon, "On the different manifestations of particle accumulation structures (pas) in thermocapillary flows", The European Physical Journal Special Topics 219, 59-69 (2013).

${ }^{44}$ D. E. Melnikov, T. Watanabe, T. Matsugase, I. Ueno, and V. Shevtsova, "Experimental Study on Formation of Particle Accumulation Structures by a Thermocapillary Flow in a Deformable Liquid Column", Microgravity Science and Technology 26, 365-374 (2014).

${ }^{45}$ M. Lappa, S. Yasushiro, and N. Imaishi, "3d numerical simulation of on ground marangoni flow instabilities in liquid bridges of low prandtl number fluid", International Journal of Numerical Methods for Heat and Fluid Flow 13, 309-340 (2003).

${ }^{46}$ M. Lappa, R. Savino, and R. Monti, "Three-dimensional numerical simulation of marangoni instabilities in non-cylindrical liquid bridges in microgravity", International Journal of Heat and Mass Transfer 44, 1983-2003 (2001).

${ }^{47}$ M. Lappa, "Combined effect of volume and gravity on the three-dimensional flow instability in noncylindrical floating zones heated by an equatorial ring", Physics of Fluids 16, 331-343 (2004).

${ }^{48}$ F. H. Muldoon and H. C. Kuhlmann, "Different particle-accumulation structures arising from particle-boundary interactions in a liquid bridge", International Journal of Multiphase Flow 59, $145-159$ (2014).

${ }^{49}$ A. Babiano, J. H. E. Cartwright, O. Piro, and A. Provenzale, "Dynamics of a small neutrally buoyant sphere in a fluid and targeting in hamiltonian systems", Phys. Rev. Lett. 84, 5764-5767 (2000).

${ }^{50}$ A. Hjelmfelt and L. Mockros, "Motion of discrete particles in a turbulent fluid", Appl. Sci. Res. 16, 149-161 (1966).

${ }^{51}$ C. F. M. Coimbra and R. H. Rangel, "Spherical particle motion in harmonic stokes flows", AIAA Journal 39, 1673-1682 (2001). 
${ }^{52}$ C. F. M. Coimbra, D. L'Esperance, R. A. Lambert, J. D. Trolinger, and R. H. Rangel, "An experimental study on stationary history effects in high-frequency stokes flows", Journal of Fluid Mechanics 504, 353-363 (2004).

${ }^{53}$ D. Vojir and E. Michaelides, "Effect of the history term on the motion of rigid spheres in a viscous fluid", International Journal of Multiphase Flow 20, 547-556 (1994).

${ }^{54}$ O. Druzhinin and L. Ostrovsky, "The influence of basset force on particle dynamics in twodimensional flows", Physica D: Nonlinear Phenomena 76, 34-43 (1994).

${ }^{55}$ R. Issa, "Solution of the implicitly discretised fluid flow equations by operator-splitting", Journal of Computational Physics 62, 40-65 (1986).

${ }^{56}$ C. M. Rhie and W. L. Chow, "Numerical study of the turbulent flow past an airfoil with trailing edge separation", AIAA Journal 21, 1525-1532 (1983).

${ }^{57}$ M. Lappa, "Three-dimensional numerical simulation of marangoni flow instabilities in floating zones laterally heated by an equatorial ring", Physics of Fluids 15, 776-789 (2003).

${ }^{58}$ W. Hu, J. Shu, R. Zhou, and Z. Tang, "Influence of liquid bridge volume on the onset of oscillation in floating zone convection i. experiments", Journal of Crystal Growth 142, 379-384 (1994).

${ }^{59}$ H. A., N. S., and S. M., "Experimental results of oscillatory marangoni convection in a liquid bridge under normal gravity", J. Jpn. Soc. Microgravity Appl. 14, 122-129 (1997).

${ }^{60}$ H. A., S. M., O. N., K. M., M. T., and K. H., "Transition process from laminar to oscillatory marangoni convection in a liquid bridge under normal and microgravity", J. Jpn. Soc. Microgravity Appl. 14, 137-1443 (1997).

${ }^{61}$ C. Nienhüser and H. C. Kuhlmann, "Stability of thermocapillary flows in non-cylindrical liquid bridges", Journal of Fluid Mechanics 480, 333-334 (2003).

${ }^{62}$ M. Ermakov and M. Ermakova, "Linear-stability analysis of thermocapillary convection in liquid bridges with highly deformed free surface", Journal of Crystal Growth 266, Proceedings of the Fourth International Workshop on Modeling in Crystal Growth, 160-166 (2004).

${ }^{63} \mathrm{Z}$. Tang and W.-R. Hu, "Influence of liquid bridge volume on the onset of oscillation in floatingzone convection: iii. three-dimensional model", Journal of Crystal Growth 207, 239-246 (1999). 
${ }^{64}$ B.-C. Sim and A. Zebib, "Thermocapillary convection in liquid bridges with undeformable curved surfaces", Journal of Thermophysics and Heat Transfer 16, 553-561 (2002).

${ }^{65} \mathrm{Q}$. Chen and $\mathrm{W}$. Hu, "Influence of liquid bridge volume on instability of floating half zone convection", International Journal of Heat and Mass Transfer 41, 825-837 (1998).

${ }^{66}$ F. Preisser, D. Schwabe, and A. Scharmann, "Steady and oscillatory thermocapillary convection in liquid columns with free cylindrical surface", Journal of Fluid Mechanics 126, 545-567 (1983).

${ }^{67}$ L. G. Leal, Advanced transport phenomena: fluid mechanics and convective transport processes, Cambridge Series in Chemical Engineering (Cambridge University Press, 2007).

${ }^{68}$ Y. Sasaki, S. Tanaka, and H. Kawamura, "Particle accumulation structure in thermocapillary convection of small liquid bridge", Transactions of the Japan Society of Mechanical Engineers Series B 70 (2004). 